\title{
Genomic insights into host adaptation between the wheat stripe rust pathogen (Puccinia striiformis f. sp. tritici) and the barley stripe rust pathogen (Puccinia striiformis f. sp. hordei)
}

\author{
Chongjing Xia ${ }^{1}$, Meinan Wang ${ }^{1}$, Chuntao Yin ${ }^{1}$, Omar E. Cornejo ${ }^{2}$, Scot H. Hulbert ${ }^{1}$ and Xianming Chen ${ }^{1,3^{*}}$ (D)
}

\begin{abstract}
Background: Plant fungal pathogens can rapidly evolve and adapt to new environmental conditions in response to sudden changes of host populations in agro-ecosystems. However, the genomic basis of their host adaptation, especially at the forma specialis level, remains unclear.

Results: We sequenced two isolates each representing Puccinia striiformis f. sp. tritici (Pst) and P. striiformis f. sp. hordei (Psh), different formae speciales of the stripe rust fungus $P$. striiformis highly adapted to wheat and barley, respectively. The divergence of Pst and Psh, estimated to start 8.12 million years ago, has been driven by high nucleotide mutation rates. The high genomic variation within dikaryotic urediniospores of $P$. striiformis has provided raw genetic materials for genome evolution. No specific gene families have enriched in either isolate, but extensive gene loss events have occurred in both Pst and Psh after the divergence from their most recent common ancestor. A large number of isolate-specific genes were identified, with unique genomic features compared to the conserved genes, including 1) significantly shorter in length; 2) significantly less expressed; 3) significantly closer to transposable elements; and 4) redundant in pathways. The presence of specific genes in one isolate (or forma specialis) was resulted from the loss of the homologues in the other isolate (or forma specialis) by the replacements of transposable elements or losses of genomic fragments. In addition, different patterns and numbers of telomeric repeats were observed between the isolates.

Conclusions: Host adaptation of P. striiformis at the forma specialis level is a complex pathogenic trait, involving not only virulence-related genes but also other genes. Gene loss, which might be adaptive and driven by transposable element activities, provides genomic basis for host adaptation of different formae speciales of $P$. striiformis.
\end{abstract}

Keywords: Stripe rust, Puccinia striiformis, Formae speciales, Host adaptation, Comparative genomics, Gene loss, Evolution

\footnotetext{
* Correspondence: xianming.chen@ars.usda.gov

'Department of Plant Pathology, Washington State University, Pullman, WA

99164-6430, USA

${ }^{3}$ Wheat Health, Genetics, and Quality Research Unit, Agriculture Research

Service, U.S. Department of Agriculture, Pullman, WA 99164-6430, USA

Full list of author information is available at the end of the article
}

(c) The Author(s). 2018 Open Access This article is distributed under the terms of the Creative Commons Attribution 4.0 International License (http://creativecommons.org/licenses/by/4.0/), which permits unrestricted use, distribution, and reproduction in any medium, provided you give appropriate credit to the original author(s) and the source, provide a link to the Creative Commons license, and indicate if changes were made. The Creative Commons Public Domain Dedication waiver (http://creativecommons.org/publicdomain/zero/1.0/) applies to the data made available in this article, unless otherwise stated. 


\section{Background}

Filamentous plant pathogens (e.g. fungi and oomycetes) cause severe diseases of crops and threaten global food security [1]. Remarkably, the populations of many devastating pathogens have been rapidly evolving and continue to emerge or reemerge in the agrio-ecosystems [2]. Understanding their evolution will be valuable for monitoring such pathogens, and for designing strategies and methods for sustainable management. The advance of whole-genome sequencing technologies has enabled us to disentangle the genomic basis underlying the host-pathogen co-evolution and demonstrated that the 'two-speed genomes' have contributed to the rapid evolution of filamentous plant pathogens [3-5]. In the 'two-speed genome' concept, genome parts (or genomic compartments) harboring pathogenicity-related genes, which are usually conditionally dispensable, have a higher evolution rate than the essential parts of the genome. Tremendous genomic studies of filamentous plant pathogens have shown that the pathogenicity-related genes reside in such rapidly evolving genomic compartments including gene clusters [6], gene-sparse and repeat-rich regions [7], AT-rich isochores [8] and supernumerary (conditionally dispensable) chromosomes [9, 10]. While most of these studies were on pathogenicity-related genes, details of genomic basis of host adaptation in filamentous plant pathogens remain to be elucidated even though some of the pathogenicity genes were also host specificity determinants [10].

Among filamentous plant pathogens, the genus Puccinia is a group of destructive rust fungi. As obligate biotrophs, they depend on living plant cells to complete their life cycle, and are considered highly specialized on their hosts. A single Puccinia species may have different forms, with each form mainly infecting specialized genera or species of susceptible hosts but not distinguishable from other forms by morphological characters. Such forms are called formae speciales (sing. forma specialis; abbr. f. sp.). For instance, Puccinia striiformis, causing stripe (yellow) rust diseases on cereal crops and grasses, has been reported to have nine formae speciales [11]. The two most economically important forms are $P$. striiformis f. sp. tritici (Pst) and P. striiformis f. sp. hordei $(P s h)$, specialized, but not exclusively, on wheat and barley, respectively. Although their host ranges overlap on some grasses [12, 13], the two formae speciales were clearly separated into closely-related but distinct lineages based on molecular markers [13]. Formae speciales have been documented in many other devastating filamentous plant pathogen species, including Blumeria graminis causing powdery mildews on cereal crops and grasses [14], Fusarium oxysporum causing vascular wilts and blights on many plant species [15] and Sphacelotheca reiliana (also known as Sporisorium reilianum) causing head smuts of maize, sorghum and related plants [16]. Genomics studies have begun to shed light on our understanding of formae speciales. For example in $B$. graminis, comparative genomics determined that the forma specialis triticale, which is pathogenic on triticale derived from crosses between Triticum and Secale species, is a hybrid of two other formae speciales tritici and secalis [17]. However, in most cases the evolution of formae speciales within a species remains obscure. Only a few isolates of Pst but no isolates of Psh have been sequenced, and therefore, it was not clear whether Pst and Psh have divergent genomes or how they evolved into different formae speciales.

The objective of this study was to decipher the genomic basis of host adaptation of $P$. striiformis at the forma specialis level. We selected two P. striiformis isolates to represent formae speciales tritici and hordei, highly adapted to wheat and barley, respectively. We took the advantages of advanced sequencing technologies to generate reference genomes with high continuity for comparative analysis. Our data suggest that the rapidly evolving and loss-of-function genes provide the potential genomic basis for host adaption in P. striiformis.

\section{Results}

Host adaptation of $P$. striiformis

We selected isolates 93-210 of Pst and 93TX-2 of Psh to represent the wheat and barley stripe rust fungi, respectively for understanding the potential genomic basis of their host adaptation. Our pathogenicity tests showed that the Pst (93-210) and Psh (93TX-2) isolates were highly virulent to their respective hosts, but highly avirulent to the other host (Fig. 1). Moreover, Pst (93-210) was virulent to wheat lines with stripe rust resistance genes $Y r 2, Y r 17, Y r 21$ and $Y r 28$, but avirulent to all barley stripe rust differential cultivars. Similarly, Psh (93TX-2) was virulent to barley differential cultivars Topper, Emir and Abed Binder 12, but avirulent to all wheat differential lines. In summary, our pathogenicity tests showed the host adaptations of these two isolates, and confirmed their status of different formae speciales of $P$. striiformis.

\section{Genome features and comparison}

We sequenced isolates Pst (93-210) and Psh (93TX-2) using both Illumina HiSeq PE150 (>70-fold coverage) and the Pacific Biosciences (PacBio) single molecule real-time sequencing technology (>50-fold coverage). This is the first report for the genome sequence of Psh, the barley stripe rust pathogen. Both isolates were predicted to be approximately $89 \mathrm{Mb}$ in genome size and highly heterozygous (Table 1; Additional file 1: Figure S1). Both genomes contain high but slightly different percentages of repeated sequences, $36.03 \%$ in Pst (93-210) and 


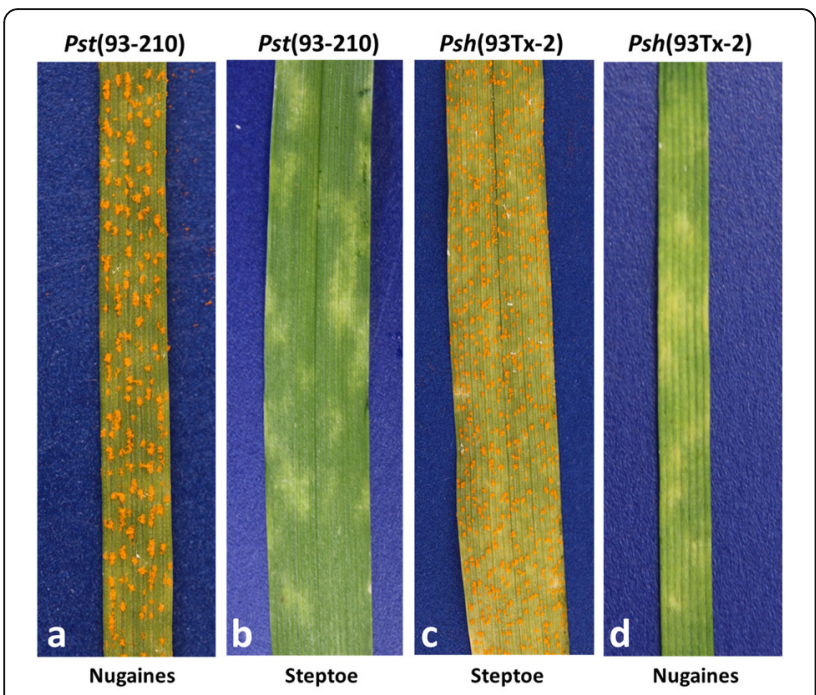

Fig. 1 Host adaptation of Puccinia striiformis. a, b Pathogenicity test of Pst (93-210) on wheat cultivar Nugaines and barley cultivar Steptoe. c, d Pathogenicity test of Psh (93TX-2) on barley cultivar Steptoe and wheat cultivar Nugaines. Note that wheat cultivar Nugaines is susceptible to all Pst isolates collected in the U.S., and barley cultivar Steptoe is susceptible to all Psh isolates collected in the U.S

$34.20 \%$ in Psh (93TX-2), which are slightly higher than those of isolates 2K-41-Yr9 (PST-78) and CYR32 but lower than that of isolate 104E 137A- [18-23]. Differences in the presence and number of unique transposable elements were observed between Pst (93-210) and Psh
(93TX-2) and also between these and other Pst isolates (Additional file 2: Table SE1) [18-23].

We predicted 16,513 and 15,976 protein-coding genes in Pst (93-210) and Psh (93TX-2), respectively. Among them, 46.26 and $45.68 \%$ were validated by the RNA sequences of these isolates obtained in this study. The annotations of the two genomes are similar in completeness, at $95.10 \%$ for Pst (93-210) and $93.00 \%$ for Psh (93TX-2), which are comparable to previously annotated Pst genomes (Additional file 3: Table S1) [18-23]. The Psh (93TX-2) genome encodes 1624 (10.96\%) predicted secreted proteins (SPs), slightly higher than the number from Pst (93-210) (1517, or 9.18\%) but within the range predicted for other Pst isolates (8.27 to 12.92\%) (Table 1). Our blastp and domain-based searches functionally annotated approximately $50 \%$ of the predicted genes: (1) 8151 and 7776 were mapped to at least one gene ontology (GO) term in Pst (93-210) and Psh (93TX-2) genomes, respectively; (2) 6450 and 6038 were found to have functionally defined homologues in the Pfam database for the two isolates, respectively; and (3) 1672 and 1131 were mapped to enzymes in the Kyoto Encyclopedia of Genes and Genomes (KEGG) database, respectively. No particular GO terms were significantly enriched in either genome. Comparisons of selected protein families also did not show apparent differences between Pst and Psh, and were also not found when wheat or barley adapted forma speciales of B. graminis were compared (Table 2; Additional file 1: Figure S2; Additional file 2: Table SE2-8) [24, 25].

Table 1 Genome statistics of Puccinia striiformis isolates Pst (93-210) and Psh (93TX-2) in comparison with previously sequenced isolates ${ }^{a}$

\begin{tabular}{|c|c|c|c|c|c|}
\hline & Pst (93-210) & Psh (93TX-2) & Pst 104E 137A- & Pst $2 \mathrm{~K}-41-\mathrm{Yr} 9$ & Pst-CY32 \\
\hline \multicolumn{6}{|l|}{ Assembly statistics } \\
\hline Genome size in contigs (bp) & $84,627,802$ & $77,368,122$ & $83,355,616$ & $79,310,008$ & $115,495,157$ \\
\hline GC-content (\%) & 44.39 & 44.40 & 44.40 & 44.43 & 44.76 \\
\hline Number of contigs & 493 & 562 & 156 & 17,295 & 10,913 \\
\hline Number of contigs $>100 \mathrm{~kb}$ & 243 & 263 & 93 & 3 & 25 \\
\hline Contig N50 & 83 & 110 & 23 & 1320 & 1434 \\
\hline Contig N50 length (bp) & 295,440 & 218,468 & $1,304,018$ & 17,361 & 21,648 \\
\hline Mean contig size (bp) & 171,659 & 137,666 & 534,331 & 4586 & 10,583 \\
\hline \multicolumn{6}{|l|}{ Annotation statistics } \\
\hline Protein-coding genes & 16,513 & 15,976 & 15,928 & 19,912 & $25288^{b}$ \\
\hline Annotation completeness ${ }^{\complement}(\%)$ & 95.10 & 93.00 & 97.60 & 98.60 & $94^{\mathrm{b}}$ \\
\hline Secreted proteins & 1517 & 1624 & 2059 & 2056 & $2092^{b}$ \\
\hline tRNA genes & 648 & 626 & 636 & 668 & 862 \\
\hline Transposable elements (Mb) & 30.46 & 26.41 & 31.08 & 24.87 & 37.68 \\
\hline Transposable elements (\%) & 36.03 & 34.2 & 37.28 & 31.35 & 32.62 \\
\hline
\end{tabular}

${ }^{\mathrm{a}}$ Statistics were calculated using the latest references and annotations except noted elsewhere

${ }^{b}$ Data was directly retrieved from Zheng et al. (2013) as the annotation of Pst-CY32 was not publicly available at the time of writing this paper

'Program BUSCO [95] was used to evaluate the annotation completeness of assembled genomes 
Table 2 Comparison of specific protein families of Puccinia striiformis isolates Pst (93-210) and Psh (93TX-2) with Blumaria graminis isolates Bgt and $B g h^{a}$

\begin{tabular}{lllll}
\hline & Pst (93-210) & Psh (93TX-2) & Bgt & Bgh \\
\hline CAZymes & 369 & 349 & 182 & 184 \\
Cytochrome P450 & 21 & 19 & 10 & 9 \\
Genes involved in & 748 & 693 & 1059 & 1030 \\
pathogen-host interaction & & & & \\
Hydrolases & 878 & 807 & 564 & 594 \\
Isomerases & 59 & 63 & 52 & 57 \\
Ligases & 87 & 89 & 73 & 78 \\
Lyases & 68 & 56 & 59 & 60 \\
Mating-related & 7 & 4 & 4 & 3 \\
Oxidoreductases & 192 & 177 & 170 & 174 \\
Proteases & 1160 & 1056 & 989 & 1025 \\
Secondary metabolites & 29 & 33 & 18 & 18 \\
Transcription factors & 104 & 88 & 73 & 76 \\
Transferases & 427 & 397 & 376 & 386 \\
Transporters & 407 & 381 & 466 & 407 \\
\hline
\end{tabular}

${ }^{\mathrm{a}}$ The data of Pst (93-210) and Psh (93TX-2) were generated in this study and the data of Bgt and Bgh were from Wicker et al. 2013 and Spanu et al. 2010

We observed extensive large syntenic blocks of nucleotides shared among the Pst (93-210), Psh (93TX-2) and Pst (104E 137A-) genomes [23]. The mean lengths of syntenic blocks are 12.64 and $13.06 \mathrm{~kb}$ when comparing Psh (93TX-2) to Pst (93-210) and Pst (104E 137A-), respectively. These mean lengths are shorter (approximately 66\%) than that of the syntenic blocks between the two Pst isolates (19.55 kb) (Additional file 1: Figure S3). Similarly, we observed slightly lower mean nucleotide identities in the Psh-Pst isolate comparisons (97.30 and $97.32 \%$ ) than the mean nucleotide identity between Pst and Pst isolates (97.59\%). These data suggested that isolates within a forma specialis are more closely related than isolates of different formae speciales, and therefore, the data confirmed the taxonomic relationships between Pst and Psh. By reciprocal mapping of the Illumina reads to both genomes, we did not detect large deleted regions but only mosaics of small regions $(<50 \mathrm{~kb})$ that were present in one reference genome but missing in the other forma specialis (Fig. 2a; Additional file 1: Figure S4). The total lengths of form-specific regions were 988 and $495 \mathrm{~kb}$, harboring 87 and 48 genes, in Pst (93-210) and Psh (93TX-2), respectively. Besides the high degree of similarity at the nucleotide level, Pst (93-210) and Psh (93TX-2) also share large collinear orthologous blocks (Fig. 2b; Additional file 1: Figure S5). We observed that only $10,769(63.79 \%)$ of the Pst (93-210) predicted proteins are homologous with 10,417 (65.20\%) of the Psh (93TX-2) proteins (see Methods).

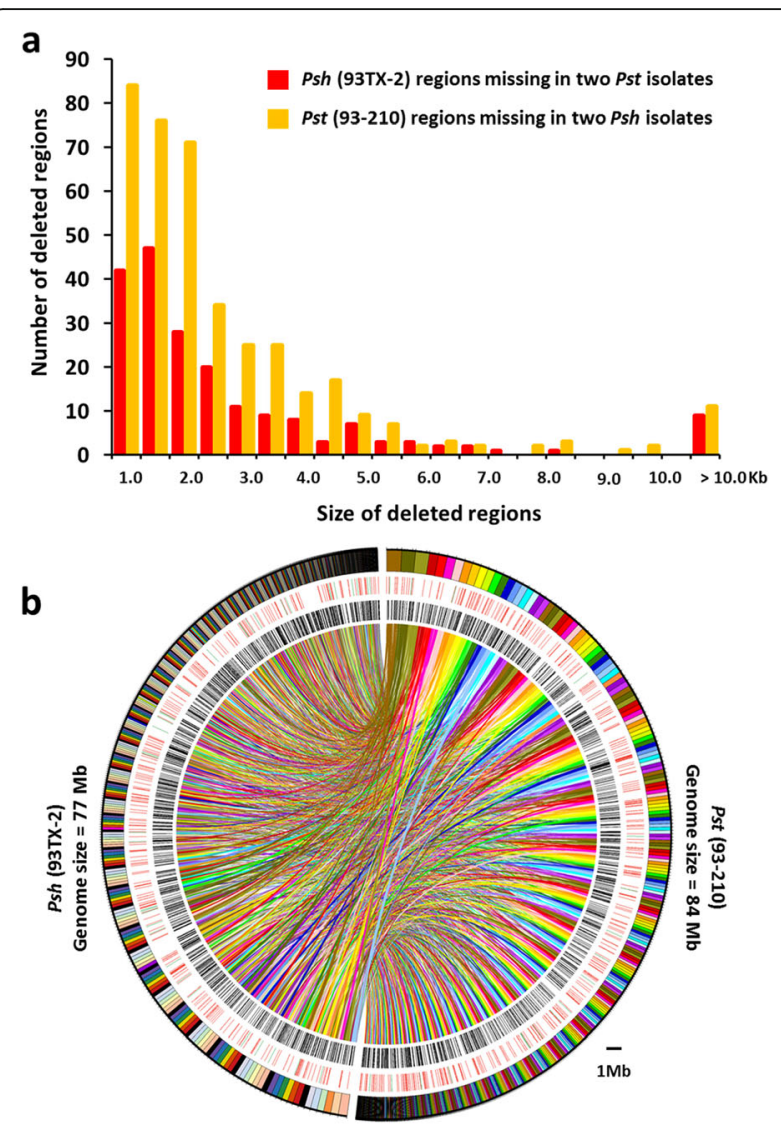

Fig. 2 Genome comparison and synteny analysis. a Distributions of various sizes of deleted genome regions presented in isolate Psh (93TX-1) but missing in two Pst isolates (93-210 and 2K-41-Yr9) and those presented in isolate Pst (93-210) but missing in two Psh isolates (93TX-2 and 04-051) of Puccinia striiformis. b Genome-wide syntenic relationships between Pst (93-210) and Psh (93TX-2). The right and left in the Circos diagram are Pst (93-210) and Psh (93TX-2) genomes, respectively. Each bar in the outer layer represents one contig, organized from largest to shortest clockwise. The second layer shows genes involved in the evolution of gene families. Green and red bars are genes involved in family gain and loss, respectively. Black bars in the third layer represent genes specific to each isolate and without orthologs in the other 15 tested plant fungal pathogens. The colored links in the inner layer represent homologous gene pairs between Pst (93-210) and Psh (93TX-2)

Using the OrthoFinder program, we assigned 175,188 protein sequences (89.4\% of total) to 20,696 orthogroups. There were 2160 orthogroups with all fungal species present and 452 of these consisted entirely of single-copy genes. In total, 15,641 (94.7\%) of Pst (93-210) proteins and 15,009 (93.9\%) of Psh (93TX-2) proteins were assigned to 9165 and 9044 orthogroups, respectively. Of these, 10,769 (63.79\%) of Pst (93-210) proteins and 10,417 (65.20\%) of Psh (93TX-2) proteins were homologous and assigned to 9316 orthogroups. This indicated that 4872 $(15,641-10,769)$ of Pst (93-210) proteins and 4592 $(15,009-10,417)$ of Psh (93TX-2) proteins had orthologues 
in other fungi, but not in Psh (93TX-2) or Pst (93-210), respectively.

In addition to nuclear genomes, we also sequenced the complete mitochondrial (mt) genomes of Pst (93-210) and Psh (93TX-2) (Additional file 1: Figure S6; Additional file 3: Tables S2-S3). No difference was observed between the Pst and Psh genomes at the genic and structural levels.

\section{Divergence and evolution of Pst and Psh}

Based on the substitutions of 452 single-copy homologs in 16 selected plant pathogenic fungi, we estimated that Pst and Psh diverged approximately 8.12 million years ago (Fig. 3a), several million years after the divergence of their hosts (10-15 million years ago) [26, 27]. Our findings also indicated that the two formae speciales of $P$. striiformis were diverged around the time of the divergence of Bgt and Bgh (9.22 million years), which are also specialized on wheat and barley, respectively [24]. Based on our estimated divergence time and the substitutions of 5611 pairs of single-copy gene homologs between Pst (93-210) and Psh (93TX-2), we further estimated the nucleotide mutation rate as $2.0 \times 10^{-8}$ per base per year, which is $\sim 40$ fold higher than that of humans $\left(0.033-0.047 \times 10^{-8}\right)$ [28]. The high mutation rate could provide the ability to generate genomic variations in $P$. striiformis and accelerate adaptations to different plant hosts by its different formae speciales.

We detected a high frequency of heterozygosity between the two nuclei within each isolate at 6-7 SNPs per kb (Table 3), which is comparable to another US Pst isolate [19]. Reciprocal mapping of the Illumina reads to each reference showed that the inter-isolate SNP density was 1.20 -fold higher than that of intra-isolate. We also observed both high intra- and inter-isolate InDel densities at $\sim 2$ InDels per kb. Remarkably, the functional prediction indicated that the intra-isolate mutations changed many protein-coding genes, 5909 in Pst (93-210) and 5659 in Psh (93TX-2) (Additional file 3: Tables S4 and S5). Among these, 1920 and 2147 genes were affected by SNPs in Pst (93-210) and Psh (93TX-2); and the InDels affected 5364 and 5174 genes in these isolates, respectively. The affected genes are diverse in predicted function, including transporters and transcription factors (TFs) (Additional file 3: Table S5). Among the transporters, there are two superfamilies enriched in genes impacted by mutations, the P-type ATPase superfamily [16 genes in Pst (93-210) and 14 genes in Psh (93TX-2)] and the protein kinase superfamily [17 in Pst (93-210) and 18 in Psh (93TX-2)] (Additional file 2: Table SE8). The proteins in the former superfamily are essential for ion-transporting; while some proteins in the latter superfamily are presumed to contribute to signal transduction and fungal pathogenesis. Remarkably in TFs, most of the heat shock factors [7 out of
8 in Pst (93-210) and 6 out of 10 in Psh (93TX-2)] were highly impacted by mutations, while all remaining TFs had moderate impacts (e.g., codon insertions and deletions, non-synonymous substitutions). Our data indicated that the mutations between two nuclei of a $P$. striiformis urediniospore have contributed to the intra-isolate genetic diversity and provided the evolutionary potential for host adaptation.

We used $\mathrm{dN} / \mathrm{dS}$ analysis to detect genes under positive selection during the evolution of $P$. striiformis. We did not observe significant differences $(p=0.92$, two-tailed student t-test) between the $\mathrm{dN} / \mathrm{dS}$ ratio distributions of SP and non-SP genes $(0.7733 \pm 0.56$ and $0.7759 \pm 0.62$, respectively) (Fig. 3b). Among the 5611 single-copy protein genes that had homologues in Pst (93-210) and Psh (93Tx-2), 1125 were identical and 4486 were different between the two isolates. When the $\mathrm{dN} / \mathrm{dS}$ ratios of the 4486 genes were compared, $28.33 \%$ (1271) had dN/dS ratios greater than 1.0, indicating positive selection. Together, these data suggested that a large number of both SP- and non-SP-coding genes have been positively selected during the Pst and Psh divergence. We found that genes under purifying selections are enriched for specific families except those with SP genes (Additional file 3: Table S5). For instance, $3.76 \%$ of the genes under purifying selections are transporter-coding genes, with only $1.25 \%$ of the genes in this family predicted to be under positive selection and this trend was observed in most other protein families. However, more SP-coding genes were under positive selection (11.48\%) than those under purifying selection $(9.95 \%)$. The SP genes under positive selection are involved in diverse pathways ( 2 in metabolic pathways, 4 in translations and protein processing in endoplasmic reticula and 2 in signal transduction).

\section{Loss of gene families}

We examined the evolutions of the gene families of Pst (93-210) and Psh (93TX-2) in 16 plant pathogenic fungi (Additional file 2: Tables SE9 and SE10). We observed extensive gene families gained at internodes, while gene family losses were much more prominent at externodes on our reconstructed phylogenetic tree (Fig. 4a). This indicates that gene family losses have occurred during speciation or formation of formae speciales in all of the plant pathogenic fungi we examined. We noted that the topology inferred from the presence or absence of gene families (Fig. 4a) was different from that inferred from amino acid substitutions (Fig. 3a), indicating that gene family evolution was independent of substitution events. Particularly for Pst (93-210) and Psh (93TX-2), five gene families were gained in each genome after the divergence from their most recent common ancestor (Fig. 4a). The gained five gene families (containing 21 genes) in Pst (93-210) have unknown functions since they do not 

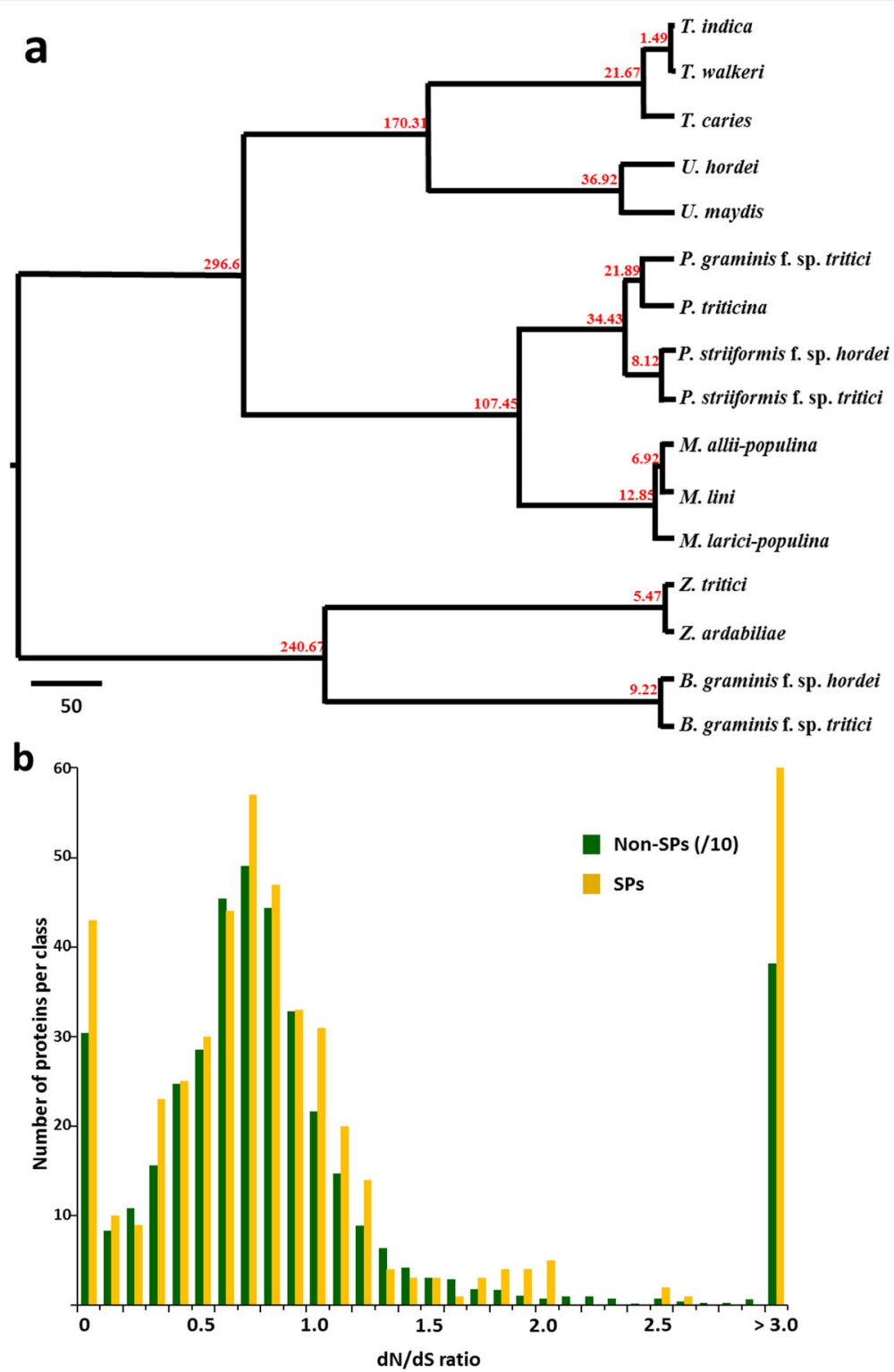

Fig. 3 Divergence and dN/dS analysis. a Estimation of the divergence times of selected fungal plant pathogens. The ultrametric tree was inferred from 439 single-copy orthologs (predicted coding sequences). Divergence times were estimated using r8s and calibrated on the 452 million years divergence between Ascomycetes and Basidiomycetes. B: Blumeria; M: Melampsora; P: Puccinia; T: Tilletia; U: Ustilago; and Z: Zymoseptoria. Z. tritici traditionally called Mycosphaerella graminicola. b Comparison of 4486 pairs of Pst (93-210) and Psh (93TX-2) single-copy homologs. The X axis represents the ratio of nonsynonymous to synonymous substitutions (dN/dS) for all gene pairs, and the $Y$ axis represents the number of gene pairs in each dN/dS range. The orange bars represent the 478 gene pairs of Pst (93-210) and Psh (93TX-2) single-copy homologs encoding secreted protein (SP) genes, and the green bars represent the 4008 non-SP-coding gene pairs. For better visibility, the numbers for non-SP genes were divided by 10

contain any Pfam domains or GO terms (Table 4). Only one gained gene in Pst (93-210) encodes a predicted SP. The functions of most (36 of 39 genes) of the gained five families in Psh (93TX-2) were also unknown. In contrast, both Pst (93-210) and Psh (93TX-2) experienced more gene family losses (190 and 311 families, respectively) after the divergence from the most recent common ancestor. Generally, Psh (93TX-2) experienced more extensive losses in the numbers of gene families and individual genes. In either Pst (93-201) or Psh (93Tx-2) genome, some genes encoding predicted SPs were mentioned in one of the genomes and expressed only in the genome keeping the genes based on our RNA-Seq analysis. Psh (93TX-2) lost more KEGG pathway-related genes $(30.9 \%$, or 141 out of 456) than Pst (93-210) (20.0\%, or 65 out of 325) (Additional file 2: Table SE11). 
Table 3 Summary of intra- and inter-isolate variations of Puccinia striiformis isolates Pst (93-210) and Psh (93TX-2)

\begin{tabular}{lllll}
\hline Polymorphism & Pst (93-210) & & & Psh (93TX-2) \\
\cline { 2 - 3 } & Pst (93-210) & Psh (93TX-2) & & Psh (93 (93-210) \\
\hline SNPs & 520,730 & 629,641 & 684,747 & 563,165 \\
Total SNPs & $6.42 \pm 11.80 / \mathrm{kb}$ & $7.71 \pm 12.80 / \mathrm{kb}$ & $9.16 \pm 14.05 / \mathrm{kb}$ & $7.58 \pm 12.68 / \mathrm{kb}$ \\
SNP density & & & & 156,529 \\
InDels & 152,392 & 150,289 & 159,457 & $2.19 \pm 4.18 / \mathrm{kb}$ \\
Total InDels & $1.97 \pm 4.07 / \mathrm{kb}$ & $1.93 \pm 3.88 / \mathrm{kb}$ & $2.21 \pm 4.07 / \mathrm{kb}$ & \\
InDel density & & & & \\
\hline
\end{tabular}

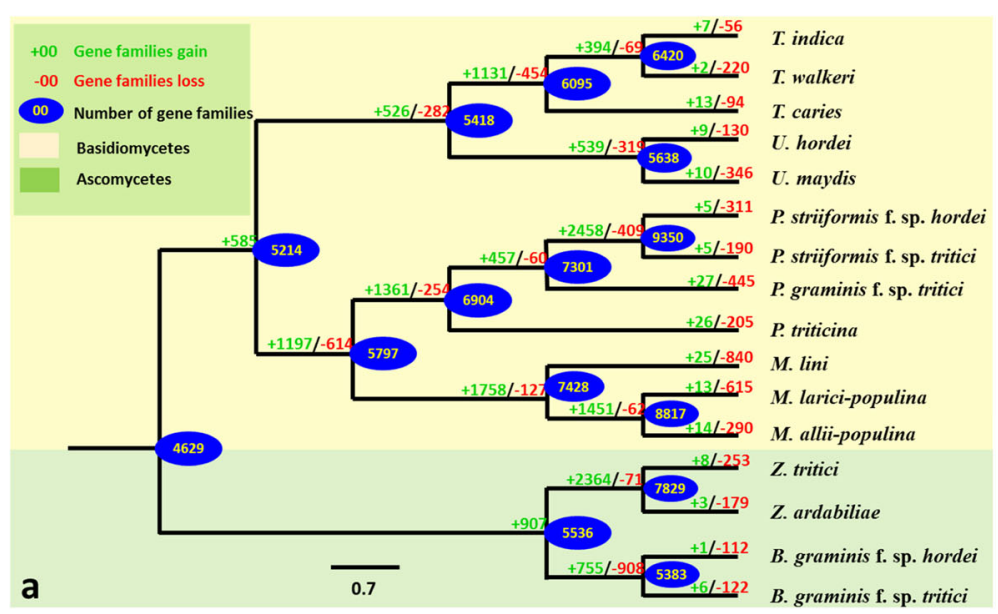

b

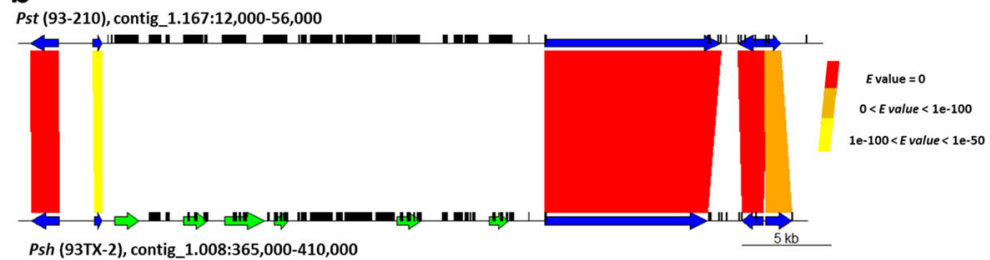

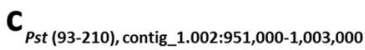

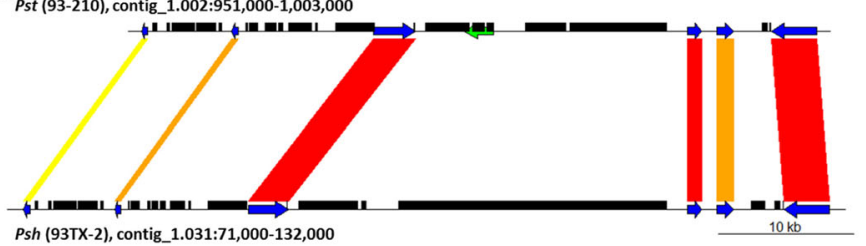

Fig. 4 Rapidly evolving gene families in Puccinia striiformis. a Gene family evolution in 16 fungal plant pathogen genomes. Protein families were assigned using OrthoFinder. The topologies and numbers of gene family gains and losses were estimated using the DOLLOP program from PHYLIP. The positive and negative numbers on the branches represent the numbers of gene families gained and lost, respectively, during the evolution compared to the putative pan-proteome. The numbers in blue ovals correspond to the inferred ancestral protein families along each lineage. b, c Chromosomal synteny of two representative regions harboring genes involved in loss events. Blue arrows represent conserved genes, while predicted genes involved in loss events are shown in green and black blocks represent homology to known transposable element families. Yellow to red shades indicate the levels of sequence similarity based on the blast $E$-values 
Table 4 Summary of gene family evolution in Puccinia striiformis isolates Pst (93-210) and Psh (93TX-2)

\begin{tabular}{|c|c|c|c|c|c|c|}
\hline & \multicolumn{3}{|c|}{ Pst (93-210) } & \multicolumn{3}{|c|}{ Psh (93TX-2) } \\
\hline & Gain & Loss & Specific & Gain & Loss & Specific \\
\hline No. of families & 5 & 190 & - & 5 & 311 & - \\
\hline No. of genes & 21 & 325 & 861 & 39 & 456 & 955 \\
\hline No. of SP-coding genes & 1 & 14 & 61 & 0 & 34 & 84 \\
\hline No. of gene expressed & 0 & 147 & 120 & 2 & 253 & 155 \\
\hline \multicolumn{7}{|l|}{ Enzymes } \\
\hline Hydrolases & 0 & 13 & 28 & 0 & 39 & 18 \\
\hline Isomerases & 0 & 0 & 0 & 0 & 1 & 4 \\
\hline Ligases & 0 & 4 & 1 & 0 & 1 & 8 \\
\hline Lyases & 0 & 1 & 1 & 0 & 6 & 0 \\
\hline Oxidoreductases & 0 & 2 & 6 & 0 & 8 & 1 \\
\hline Transferases & 0 & 6 & 10 & 0 & 8 & 17 \\
\hline \multicolumn{7}{|l|}{ GO terms } \\
\hline Total proteins with GO & 0 & 155 & 147 & 4 & 268 & 183 \\
\hline Molecular function & 0 & 73 & 186 & 4 & 156 & 229 \\
\hline Biological pathway & 0 & 106 & 185 & 0 & 187 & 275 \\
\hline Cellular component & 0 & 60 & 115 & 0 & 118 & 185 \\
\hline
\end{tabular}

The GO term annotation suggested that the missing proteins are involved in diverse functions in both genomes. However, we did not detect significant enrichment in GO terms in the missing proteins compared to the rest of the proteins in both genomes. Taking together, both Pst (93210) and Psh (93TX-2) lost many functional genes.

The genes involved in loss events are randomly dispersed along the contigs (Fig. 2b), with only few small clusters (up to six genes). As an example, one such cluster is shown in Fig. 4b, in which six genes were lost in the Pst (93-210) genome but present in the corresponding region in the Psh (93TX-2) genome. The upper panel in Fig. 4b shows that the corresponding regions of the six lost genes are replaced by transposable elements (TEs), suggesting the effects of TEs on the gene loss events. Remarkably, all these six genes with unknown functions are expressed in Psh (93TX-2), but not in Pst (93-210). Fig. 4c shows a special case in which most of the lost genes are singletons scattered in highly syntenic regions. Even so, the associations of gene losses with TE involvements are not uncommon (Fig. 4c). In some cases [161 in Pst (93-210) and 193 in Psh (93TX-2)], TE fragments are inserted in the lost genes.

\section{Rapidly evolving isolate-specific genes}

Besides the high mutation rates and pervasive gene losses, we also noticed the existence of a large number of protein-coding genes that are species- (or forma specialis-) specific among the 16 fungal genomes we compared (Additional file 2: Table SE10; Methods).
Particularly, 861 of the Pst (93-210) and 955 of the Psh (93TX-2) proteins do not have any orthologs in the other 14 fungi or the NCBI database, and therefore are exclusively present in Pst (93-210) and Psh (93TX-2), respectively. Their absence in the other haplotigs due to polymorphisms between two nuclei [23] in a dikaryon was also confirmed (Methods). Indeed, 381 [out of 861 in Pst (93-210)] and 341 [out of 955 in Psh (93TX-2)] proteins had significant hits in the protein database, and all significant hits were within genus Puccinia [mainly the 2 K-41-Yr9 (PST-78) isolate] [20]. The absence of these genes in Pst (93-210) or Psh (93TX-2), but presented in other Pst isolates, indicated them actually isolate-specific. However, the majority of the isolate-specific genes, $56 \%$ (480 of the 861 genes) in Pst (93-210) and 64\% (614 of the 955) in Psh (93TX-2), did not have significant hits in the protein database, suggesting that these non-hit genes could be forma specialis specific. Taking together, these isolate-specific genes are rapidly evolving after speciation within the Puccinia genus.

The isolate-specific genes had a mean of $876 \mathrm{bp}$ in length and were significantly shorter than the mean of conserved genes $[p=1.72 \mathrm{e}-59$ for Pst (93-210) and 4.59e-56 for Psh (93TX-2); two-tailed student $t$-test] (Fig. 5a, b). The specific genes in both isolates had significantly fewer RNA sequencing reads than the conserved genes $[p=6.01 \mathrm{e}-48$ for Pst $(93-210)$ and 6.17e-40 for Psh (93TX-2), two-tailed student $t$-test] (Fig. 5c, d). Indeed, no transcripts were identified by the RNA-Seq analysis for 65\% [557 out of 861 in Pst (93-210), and 621 out of 955 in Psh (93TX-2)] of the isolate-specific genes $(\mathrm{FPKM}=0)$; whereas all of the conserved genes were expressed. We also investigated the distances of the isolate-specific genes to the closest TEs. In Pst (93210), the mean distances of isolate-specific genes to the closest TEs were $1612 \mathrm{bp}$ at the $5^{\prime}$ ends and $1665 \mathrm{bp}$ at the 3' ends, and these distances were significantly shorter ( $p=2.57 \mathrm{e}-13$ and $1.61 \mathrm{e}-15)$ than those of the conserved genes, 3323 and 3560 bp, respectively (Fig. 5e, f). Similarly, the significantly different distances between isolate-specific genes and conserved genes to the closest TEs were also observed in Psh (93TX-2) (Fig. 5g, h). Specifically, we noticed that 201 (out of 861) in Pst (93-210) and 219 (out of 955) genes in Psh (93TX-2) were interrupted by TEs at the $5^{\prime}$ and/or 3' ends. Two examples of the relationship between isolate-specific genes and TEs are shown in Fig. 6a, b.

Given the significant low level of expression, we hypothesize that the rapidly evolving isolate-specific genes may be dispensable in biological functions. By searching the KEGG GENES database, 91.8\% (790 out of 861) in Pst (93-210) and 90.7\% (866 out of 955) in Psh $(93 \mathrm{Tx}-2)$ of isolate-specific genes could not be assigned to any KEGG pathways (Additional file 2: Table SE11). 

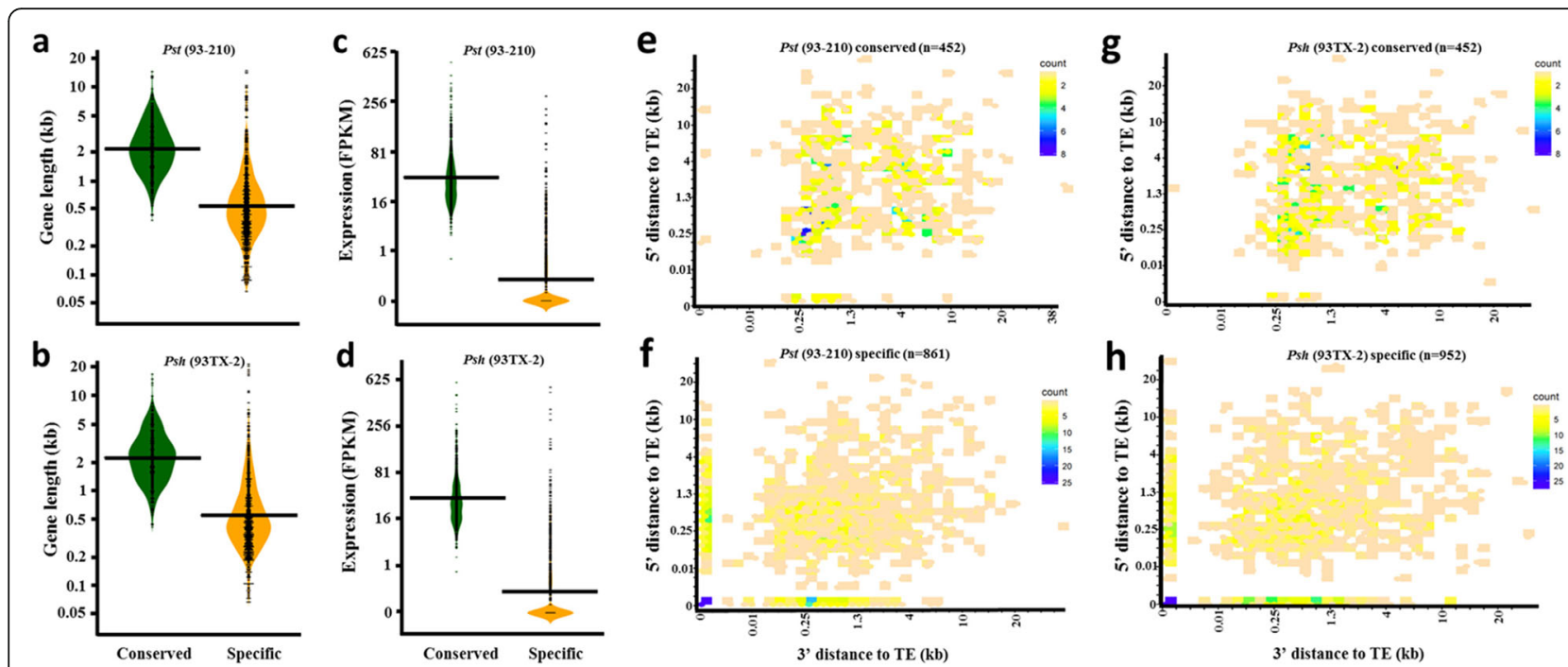

Fig. 5 Distinct features of isolate-specific genes compared to conserved genes. $\mathbf{a}$, $\mathbf{b}$ Gene length. $\mathbf{c}$, $\mathbf{d}$ Gene expression level normalized in FPKM units. FPKM, fragments per kilobase of transcript per million read pairs. e-h Distances of genes to their closest transposable elements (TEs) at the $5^{\prime}$ and $3^{\prime}$ ends. A large number of isolate-specific genes are interrupted by TEs, and therefore denoted as 0 bp away to the closest TEs in $\mathbf{f}$ and $\mathbf{h}$

In contrast, $80.5 \%$ (364 out of 452 ) of the highly conserved genes were successfully assigned to different KEGG pathways including basic biological processes such as genetic information processing (transcription, translation, replication and repair) and metabolic pathways (carbohydrate, amino acid, lipid and nucleotide metabolisms). More interestingly, most [66 out of 71 in Pst (93-210) and 80 out of 89 in Psh (93TX-2)] of the isolate-specific genes that could be assigned to KEGG pathways were accompanied by at least one KEGG orthologous gene, indicating that these genes are actually redundant genes in the pathway (Additional file 2: Table SE11). Moreover, we did not detect any particular GO terms that were significantly enriched in isolate-specific proteins comparing with the rest of the proteins in both Pst (93-210) and Psh (93TX-2). In summary, our data strongly supported that these rapidly evolving isolate-specific genes may be dispensable in essential functions.

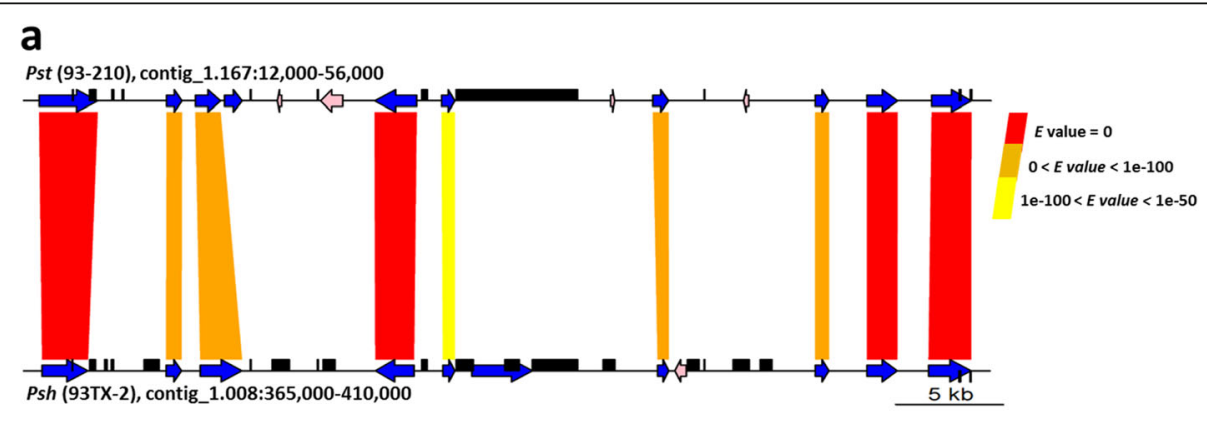

b

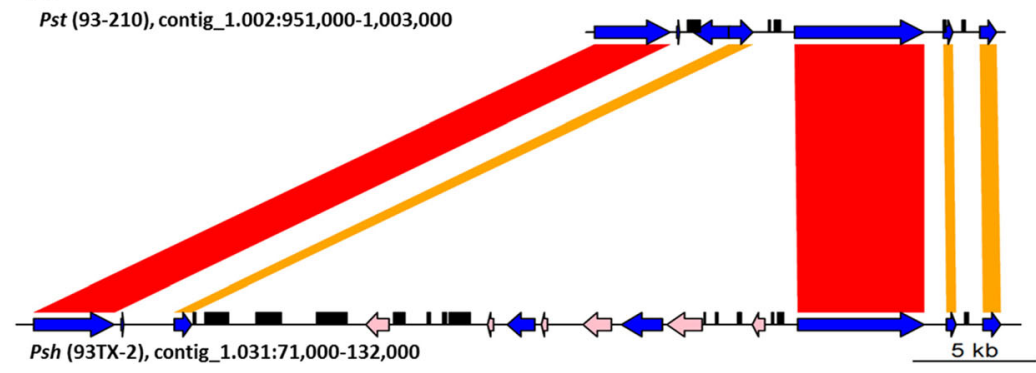

Fig. 6 Genomic environment of isolate-specific genes. See the legend of Fig. 4. Pink arrows are isolate-specific genes. The ranges of E values for the homologies in the corresponding genomic regions between Psh and Pst for both Fig. 6a and b are given in Fig. 6a 
Through reciprocal mapping of RNA-Seq reads, we identified 116 and 119 genes that were isolate-specifically expressed in Pst (93-210) and Psh (93TX-2), respectively, but silent in the other isolate (Additional file 1: Figure S7). These exclusively expressed genes were randomly dispersed throughout the genome. Among these genes, we observed slightly different numbers of SPs (22 versus 7) and carbohydrate active enzymes (5 versus 0 ) between Pst (93-210) and Psh (93TX-2). Also more exclusively expressed genes were annotated with GO terms in Pst (93-210) than Psh (93TX-2) (23 versus 13). The majority of the annotated GO terms were related to biological processes. Similar to isolate-specific genes, only $6.89 \%$ (8 out 116 ) and $3.36 \%$ (4 out of 119) were assigned to KEGG pathways, and all of them were redundant under the KEGG orthology. These results indicated that the isolate-specifically expressed genes were also dispensable and might have lost functions in the isolate in which these genes were silent.

\section{Differences in telomere repeats}

In addition to the different functional genes, Pst (93210) and Psh (93TX-2) are significantly different in the number and pattern of telomere repeats. The Pst (93-210) genome had two telomere repeat patterns (Table 5). The first repeat pattern was characterized by the presence of multiple copies (>10) of (TTTT AGGG) followed by multiple copies (> 30) of (TTAGGG); while only (TTAGGG) is presented multiple times $(>40)$ in the second repeat pattern. Pst (93-210) had both patterns in eight contigs, whereas Psh (93TX-2) had only the second pattern in five contigs. All telomere repeats were located in the ends of these contigs, indicating the completeness of the assembled telomere regions.

\section{Discussion}

In this study, we focused on deciphering the genomic basis underlying the host adaptation of filamentous plant pathogens at the forma specialis level. To this end, we generated genome references for two formae speciales in $P$. striiformis, namely $P$. striiformis f. sp. tritici using isolate Pst (93-210) that is highly adapted to wheat and P. striiformis f. sp. hordei using isolate Psh (93TX-2) that is highly adapted to barley (Fig. 1). Our genomic analyses showed that the host adaptation of formae speciales is a complex trait in P. striiformis, supported by the following two factors: i) no specific gene (or family) loss or gain could explain their adaptation, even though the two formae speciales were slightly different in specific protein family compositions (Table 2); and ii) a large number of both non-SP and SP genes were under diversifying selections, in contrast to $B$. graminis $\mathrm{f}$. sp. tritici and B. graminis f. sp. hordei in which SP genes have much higher $\mathrm{dN} / \mathrm{dS}$ ratios compared to non-SP genes [24]. However, we found the following genomic features associated with the host adaptations of Pst and Psh: i) the high genetic variations between two nuclei in a urediniospore provide raw genetic materials for genome evolution; ii) the rapidly evolving isolate-specific genes are dispensable and subject to functional loss; iii) gene loss may be adaptive during the host adaptation; and iv) transposable elements accelerate the evolution of host adaptation by involving in gene loss.

High genomic variations within dikaryotic urediniospores High heterozygosity as a function of genetic variation within dikaryotic urediniospores has been observed both in individual isolates and at the population level in $P$. striiformis [19, 22, 29, 30]. The origin of the heterozygosity remains unclear, e.g. whether via plasmogamy of two compatible but genetically distant haploid nuclei, spontaneous mutation or both. A large proportion of heterozygous genome regions and genes shared between Pst (93-210) and Psh (93TX-2) in this study (data not shown) indicates that both haplotypes and alleles had existed before the divergence of the two formae speciales. The spontaneous mutations might occur during and/or after the divergence. Regardless of the origin of the heterozygosity, our study clearly demonstrated that many of the genes were highly affected by mutations (Additional file 3: Tables S4 and S5). The impacts of the mutations might be two-sided. For instance, some mutations caused gene losses through interrupting start codons; while other mutations might create new genes through gaining stop codons. Through these ways, an isolate is able to obtain new gene functions by changing one allele and keeping the other allele functional. This can be supported by the recently identified $A v r S r 50$ gene in the wheat stem rust pathogen (P. graminis f. sp. tritici) in which the avirulence allele was functional while the insertion-disrupted virulence allele was not expressed [31]. This process might be particularly beneficial for $P$. striiformis in the geographic regions where only asexual stages exist [32-35]. This is consistent with our previous observation that Pst isolates with high homozygosity are less adaptive [29]. Besides mutations, Schwessinger et al. [23] recently observed another form of heterozygosity as one allele being totally lost for many genes in an Australian Pst isolate. Taking together, these evidences supported our hypothesis that high genomic variations within dikaryotic urediniospores provide raw genetic materials for the rapid genome evolution of P. striiformis.

\section{Isolate-specific genes subject to gene loss}

From protein family analysis, we observed a large number of protein-coding genes unable to assign to any families, suggesting that they are species- (or forma specialis-) 
Table 5 Telomere repeats and organization in isolates Pst (93-210) and Psh (93TX-2) genomes of Puccinia striiformis

\begin{tabular}{|c|c|c|c|c|c|c|}
\hline Contig name & Contig size (bp) & Start of telomeric array & End of telomeric array & $\begin{array}{l}\text { Size of telomeric } \\
\text { array }(b p)^{a}\end{array}$ & $\begin{array}{l}\text { Position of telomere } \\
\text { on contig }\end{array}$ & $\begin{array}{l}\text { Number of telomeric } \\
\text { repeats }\left(5^{\prime}-3^{\prime}\right)\end{array}$ \\
\hline \multicolumn{7}{|l|}{ Pst (93-210) } \\
\hline Contig1.035 & 512,194 & 511,887 & 512,194 & 307 & end & $(\mathrm{TTT} A \mathrm{AGGG})_{11}(\mathrm{TTAGGG})_{38}$ \\
\hline Contig1.074 & 318,575 & 318,319 & 318,575 & 257 & end & $(\mathrm{TTT} A \mathrm{TGG})_{17}(\mathrm{TTAGGG})_{21}$ \\
\hline Contig1.124 & 232,724 & 232,418 & 232,724 & 307 & end & $(T T T A G G G)_{16}(T T A G G G)_{32}$ \\
\hline Contig1.178 & 156,450 & 156,143 & 156,450 & 307 & end & $(T T T A G G G)_{10}(T T A G G G)_{40}$ \\
\hline Contig1.186 & 147,797 & 147,519 & 147,797 & 279 & end & $(\mathrm{TTT} A \mathrm{AGGG})_{10}(\mathrm{TTAGGG})_{35}$ \\
\hline Contig1.196 & 140,041 & 139,736 & 140,041 & 306 & end & $(T T T A G G G)_{8}(T T A G G G)_{40}$ \\
\hline Contig1.227 & 113,221 & 112,944 & 113,221 & 268 & end & $(T T T A G G G)_{10}(T T A G G G)_{33}$ \\
\hline Contig1.423 & 31,619 & 31,319 & 31,619 & 301 & end & $(\mathrm{TTT} A \mathrm{TGG})_{14}(\mathrm{TTAGGG})_{32}$ \\
\hline Contig1.026 & 542,491 & 542,248 & 542,491 & 244 & end & $(T T A G G G)_{42}$ \\
\hline Contig1.083 & 295,440 & 295,160 & 295,440 & 281 & end & $(\mathrm{TTAGGG})_{47}$ \\
\hline Contig1.087 & 289,332 & 289,039 & 289,332 & 294 & end & $(T T A G G G)_{49}$ \\
\hline Contig1.127 & 230,054 & 229,794 & 230,054 & 261 & end & $(T T A G G G)_{46}$ \\
\hline Contig1.151 & 197,251 & 197,012 & 197,251 & 240 & end & $(T T A G G G)_{40}$ \\
\hline Contig1.206 & 132,791 & 132,533 & 132,791 & 259 & end & $(T T A G G G)_{43}$ \\
\hline Contig1.212 & 128,166 & 127,952 & 128,166 & 215 & end & $(T T A G G G)_{35}$ \\
\hline Contig1.284 & 76,604 & 76,345 & 76,604 & 260 & end & $(T T A G G G)_{43}$ \\
\hline \multicolumn{7}{|l|}{ Psh (93TX-2) } \\
\hline Contig1.118 & 212,623 & 212,376 & 212,623 & 248 & end & $(\mathrm{TTAGGG})_{42}$ \\
\hline Contig1.162 & 163,479 & 163,194 & 163,479 & 286 & end & (TTAGGG) 47 \\
\hline Contig1.169 & 159,050 & 158,860 & 159,050 & 191 & end & $(T T A G G G)_{31}$ \\
\hline Contig1.300 & 84,330 & 84,093 & 84,330 & 238 & end & $(T T A G G G)_{40}$ \\
\hline Contig1.402 & 53,760 & 53,511 & 53,750 & 240 & end & $($ TTAGGG) 42 \\
\hline
\end{tabular}

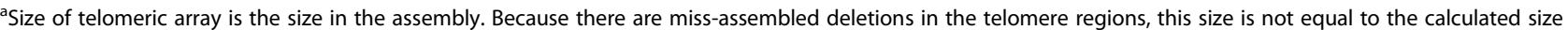
from the last column

specific among the 16 fungal genomes compared. The number of specific genes in a genome ranged from 3.2\% (210 genes in B. graminis f. sp. hordei) to $17.8 \%$ (2845 in $P$. graminis f. sp. tritici). It should be noted that the number of specific genes detected largely depends on the reference genome sets selected, meaning that the more closely related samples used, the less number of specific genes could be detected. This explains the relatively lower percentage of specific genes in B. graminis f. sp. hordei and B. graminis f. sp. tritici, and in Pst (93-210) and Psh (93TX-2) as they are different formae speciales under a species in each pair. Even so, we still were able to detect a relatively large number of specific proteins at the forma specialis level for these fungi. Particularly, 872 and 967 proteins of Pst (93-210) and Psh (93TX-2) did not have any homologous in the remaining 14 fungi genomes compared. Manual examination of these proteins in the haplotigs of assembled Pst (93-210) and Psh (93TX-2) genomes detected 11 and 12 proteins (tblastn, e-value $<1 \mathrm{e}-100$, identity $>90$ ), respectively. Therefore, we concluded that $861(=872-11)$ and $955(=967-12)$ proteins are exclusively present in Pst (93-210) and Psh (93TX-2), respectively.
We found that the FPKM values of 128 genes in Pst (93-210) was $>1.0$ when the RNA-Seq reads of Pst (93-210) were mapped, while the expression level was 0 when the RNA-Seq reads of Psh (93TX-2) were mapped. This indicated that these 128 genes were expressed during the Pst (93-210) infection, but their respective homologous genes, if they have, did not express at all in Psh (93TX-2). We also noticed that among these 128 genes, 12 genes were actually Pst (93-210)-specific genes and therefore do not have homologous genes in Psh (93TX-2). As the 12 genes have potential for developing molecular markers for monitoring the pathogen populations and studying their functions, they are present in Additional file 2: Table SE12. However, these 12 genes were excluded from the analysis for isolate-specific expression. The remaining 116 genes were considered as exclusively expressed genes in Pst (93-210), and similarly, we identified 119 genes that were exclusively expressed in Psh (93TX-2) (Additional file 2: Table SE13). The exclusively expressed genes in the Pst or Psh isolates may contribute to their host adaptation. 
Genomes of many filamentous plant pathogens harbor a large number of lineage-specific genes (Additional file 2: Table SE10; [36]), called orphan genes that lack homologues in other lineages [37]. In the present study, we identified the following three groups of isolate-specific genes in either Pst (93-210) or Psh (93TX-2). The first group includes the genes that are present in the other fungi and present in either Pst (93-210) or Psh (93TX-2) but not both. These genes have been involved in gene-loss events since the divergence of Pst and Psh (Fig. 4a). The second group contains the isolate-specific genes that are present in either Pst (93-210) or Psh (93TX-2), but absent in all other fungi. These genes have been involved in gene-gaining events since the divergence of Pst and Psh. These isolate-specific genes are distinct from the conserved genes in that they are significantly shorter in length, less expressed and closer to TEs (Fig. 5), suggesting that they are 'younger' genes and fast-evolving [37]. The third group includes the genes that are present in both Pst (93-210) and Psh (93TX-2), but exclusively expressed in either Pst (93-210) or Psh (93TX-2). In contrast to many other comparative analyses at the species or genus level with emphasis specifically on secreted effector genes [38], the SP genes identified in the present study are not enriched as specific genes for Pst (93-210) or Psh (93TX-2) (Table 4). These results indicate that the effector genes are at least not the only determinant of host specificity in these formae speciales of $P$. striiformis, and that the role of non-effector genes might be underestimated. This finding is different from the previous report of forma-specialis specific effectors in Fusarium oxysporum [39]. Similar to virulence-related genes, the specific genes in Pst (93-210) or Psh (93TX-2) are also located in plastic genomic environments enriched by TEs (Figs. 4, 5e-h, 6). The emergence of specific genes was not due to horizontal gene transfer (see Methods), and less possible by de novo evolution of genes from non-coding sequences given the relative short divergence time between Pst (93-210) and Psh (93TX-2). Instead, we speculate that the presence of specific genes in one isolate (or forma specialis) resulted from the loss of the homologues in the other isolate (or forma specialis) by replacements of TEs or loss of genomic fragments (Figs. 4b, c, 6). While functions of lineage-specific genes remain unknown, we postulate that, particularly in P. striiformis, the specific genes are dispensable and subject to gene loss. This hypothesis is supported by their lower levels or absence of expression (Fig. $5 \mathrm{c}, \mathrm{d}$, Additional file 1: Figure S7).

\section{Gene loss as putative genomic basis for host adaptation}

We summarize that gene loss is an underestimated genetic mechanism during host adaptation in filamentous plant pathogens. As we have shown above, the specific genes in Pst (93-210) and Psh (93TX-2) are the direct consequence of gene loss. Remarkably, pervasive gene-loss events after speciation were observed in all fungi we examined in the present study (Fig. 4a). This is slightly different from the previous prediction [40] since closely related plant pathogen species (and formae speciales) were selected in our study and therefore possible homologues could be distinguished at a higher power [37]. In fact, gene losses have been reported in many filamentous plant pathogens to provide evolutionary adaptive responses to changes in host and environmental conditions, and even associated with pathogen lifestyles [40]. For instance, reductions of genes encoding carbohydrate active enzymes, secondary metabolites and enzymes in nitrogen and sulfur assimilation pathways enable biotrophs adapted to plant hosts by avoiding recognition by plant defense systems $[25,41]$. Moreover, the loss of an Avr gene is an effective way of host adaptation for many plant pathogens in agro-systems in which the corresponding $R$ genes change rapidly in both space and time, e.g. AvrLm1 in Leptosphaeria maculans [42], AVR-Pita in Magnaporthe oryzae [43] and NIP1 in Rhynchosporium secalis [44]. Particularly in the recently identified AvrSr35 in P. graminis f. sp. tritici, the loss of AvrSr35 resulted from insertion of mobile element enabled avirulent isolates virulent to the $\mathrm{Sr} 35$ resistance gene [45].

We speculate that losses of some of the isolate-specific genes may be adaptive for Pst (93-210) and Psh (93TX-2) to infect their respective hosts. We provide the following reasons. Hyposensitive-reaction (HR) was observed when barley cultivar Steptoe was inoculated with most Pst isolates (Fig. 1b), suggesting that most Pst isolates have Avr_barley genes corresponding to the resistance genes in Steptoe [46]. Such Avr_barley genes in Pst must be lost or mutated in Psh, since no HR was observed when Steptoe was inoculated with all Psh isolates (Fig. 1c) [47]. Similarly, most Psh isolates should have Avr_wheat genes corresponding to the resistance genes in Nugaines (Fig. 1d), while such Avr_wheat genes in Psh must be lost or mutated in Pst since Nugaines is susceptible to all Pst isolates (Fig. 1a) [47-49]. In summary, Pst has lost the Avr_wheat genes presented in Psh, and therefore highly adapted to wheat; while Psh has lost the Avr_barley genes presented in Pst, and therefore highly adapted to barley. This is consistent with the isolate-specific genes identified in Pst (93-210) and Psh (93TX-2). Further studies are needed to characterize such genes from the hundreds of genes specific to the isolates identified in the present study.

Mutations in protein-coding DNA sequences are classified as either synonymous when they do not change the encoded amino acids, or nonsynonymous when they can change the amino acids. Since these two substitutions are under different selective pressures, the 
comparison of synonymous and nonsynonymous substitution rates ( $\mathrm{dN}$ and $\mathrm{dS}$, respectively) between paired protein-coding DNA sequences can indicate the action of natural selection on the proteins. We found that a large number of both SP- and non-SP-coding genes were positively selected during the Pst and Psh divergence and that genes under purifying selections are enriched for specific families. More SP-coding genes were under positive selection than those under purifying selection. The putative SP genes under positive selection are involved in diverse pathways. These results suggest positive selection for some of the SP genes, which may have contributed to the adaptations to different cereal crops.

\section{Differences in telomeres}

The numbers and patterns of telomere repeats in the Pst (93-210) and Psh (93TX-2) suggest different chromosomal structures between these two isolates or formae speciales. To our knowledge, this is the first report of telomere repeats in rust fungi. The repeat TTAGGG is the same as the telomeric repeat reported in vertebrates and some filamentous fungi, while TTTTAGGG is the same as the repeat in green algae (Chlamydomonas spp.) $[50,51]$. Eight contigs containing telomere repeats of $(\text { TTTTAGGG) })_{n}(\text { TTAGGG) })_{n}$ and eight contigs containing telomere repeats of (TTAGGG) $)_{\mathrm{n}}$ were found in Pst (93-210), whereas only five contigs containing (TTAG GG) $)_{n}$ were found in Psh (93Tx-2). It is not clear whether the different numbers of contigs containing telomeric repeats indicate different numbers of chromosomes in these isolates. Based on the base number of 6 chromosomes and the dikaryotic number of 12 chromosomes in dikaryotic urediniospores of $P$. striiformis previously determined by cytological and linkage analyses $[52,53]$, the five pairs of telomeric regions observed in Psh (93TX-2) indicate that the six chromosomes in one nucleus have identical numbers of the repeat in both telomeric regions of each chromosome and two of the chromosomes have identical numbers of the repeat. In contrast, the eight pairs of different repeats observed in Pst (93-210) indicate that each of the six chromosomes have different repeats in the telomeric regions [one containing (TTAG $\mathrm{GG})_{\mathrm{n}}$ and one containing (TTTTAGGG) ${ }_{\mathrm{n}}($ TTAGGG) and the two nuclei in a urediniospore have different sets of chromosomes. The differences in telomeric regions reflect chromosomal changes during the divergence of these two isolates and further work needs to be done to determine if the different numbers and patterns of telomeric repeats are conserved within each of the formae speciales. As the number of telomere repeats is associated to aging, disease and environment in humans and other vertebrates [54], the different telomeric repeats resulting from evolution may contribute to host and/or environment adaptations.

\section{Evolution of host adaptation of $P$. striiformis to wheat and barley}

Based on our genomics analyses of Pst and Psh genomes, we propose a simplified evolutionary scenario for the host adaptation of these two formae speciales, as shown in Fig. 7. The most recent common ancestor of Puccinia spp. experienced extensive gene-family gains and expansions (Fig. 4a) [36], accompanied by host jumps and shifts [55]. Then the divergence of formae speciales occurred several million years after the divergence of wheat and barley at 11.6 Mya (Fig. 3a) [27]. The divergence of Pst and Psh were concurrent with mutations (Table 3; Additional file 3: Tables S4 and S5), sexual recombinations [22], somatic recombinations [56] and other evolutionary forces [57], leading to extensive gene losses and occasionally gene gains (Fig. 4a), which provide the genomic basis for host adaptation at the formae speciales level. These evolutionary processes are accompanied and accelerated by massive TE activities as reported for other fungi [4]. We further postulate that the host adaptation is an ongoing evolutionary process and the formae speciales in $P$. striiformis may have incomplete reproductive isolation in agro-ecosystems.

\section{Conclusions}

In this study, we used comparative genomics and analyzed genomes of two isolates, Pst (93-210) and Psh (93TX-2) that are highly adapted to wheat and barley, respectively, to represent two economically important formae speciales of P. striiformis. Based on our analyses, we conclude that i) host adaptation at the forma specialis level is a complex trait, involving not only virulence-related genes but also other genes; ii) gene loss, along with other evolutionary processes, provides the genomic basis for host adaptation in P. striiformis; and iii) the evolution of host adaptation is accompanied and driven by TE activities. In addition, changes in telomeric regions may contribute to host adaptation. Our results suggest that gene loss is more important than expected in the evolution of host adaptation in filamentous plant pathogens, at least at the forma specialis level.

\section{Methods \\ Pathogenicity test}

We selected two isolates, Pst (93-210) and Psh (93TX-2) that were collected from Montana and Texas, respectively in 1993 in the US, to represent wheat and barley stripe rust pathogens, respectively [13]. We first determined their host adaptation using pathogenicity tests. For both Pst (93-210) and Psh (93TX-2) isolates, fresh urediniospores were used to inoculate both Nugaines and Steptoe. Nugaines is a wheat cultivar and susceptible to all Pst isolates collected in the US, whereas Steptoe is 


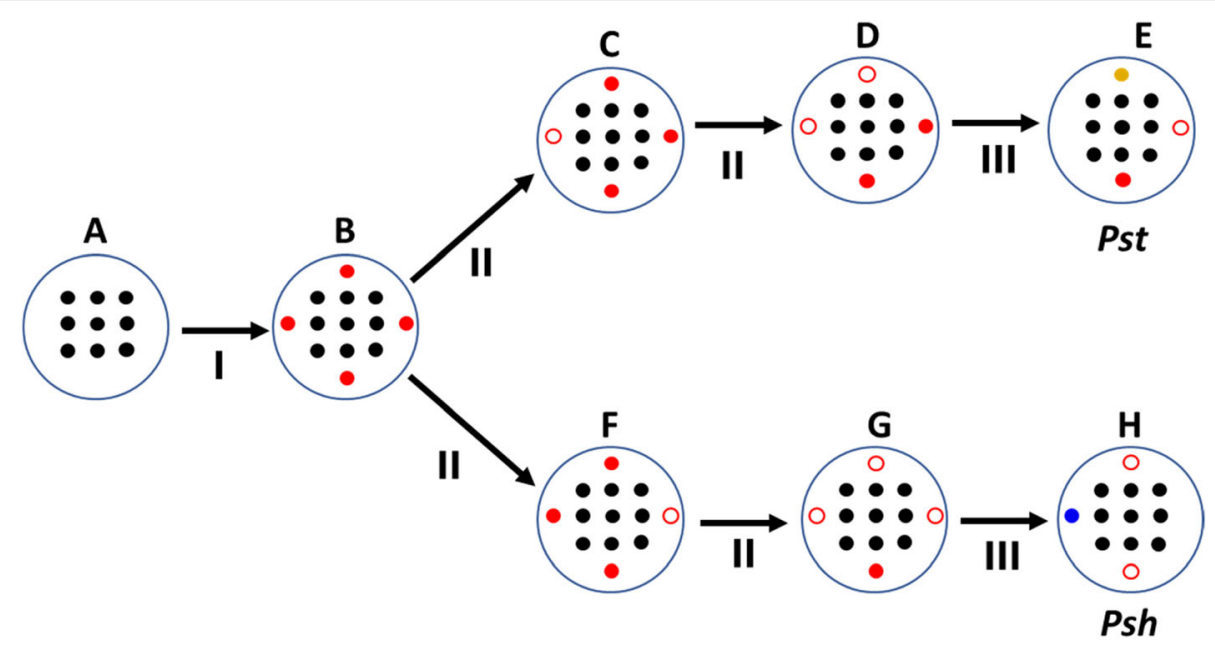

Fig. 7 A simplified evolutionary scenario for the host adaptation of Puccinia striiformis f. sp. tritici (Pst) and P. striiformis f. sp. hordei (Psh). a-h: genomes; $\mathbf{a}$, the most recent common ancestor of Puccinia spp.; $\mathbf{b}$, the most recent common ancestor of $P$. striiformis; $\mathbf{c}-\mathbf{d}$ and $\mathbf{f}-\mathbf{g}$, intermediate ancestor of Pst (e), and Psh (h), respectively. I-III: evolutionary processes; I, gene family gains and expansions during speciation; II, gene losses; III, gene losses, and/or gains, host adaptation. Black circles, conserved genes; colored circles, rapid evolving genes; solid circles, functional genes; and empty circles, loss-of-function genes

a barley cultivar and susceptible to all Psh isolates collected in the US [13, 47-49, 53]. We followed an inoculation procedure described previously [58]. Virulence phenotypes were recorded, and photos were taken 15 days after inoculation. The two isolates were also tested on the 18 wheat $Y r$ single-gene lines used to differentiate Pst races [58] and 12 barley cultivars used to differentiate Psh races [47].

\section{Genome sequencing and read preparation}

Genomic DNA was extracted from urediniospores using the CTAB method [59]. A DNA library with fragment sizes of approximately 150 bp was prepared and sequenced using the Illumina HiSeq PE 150 technology. Meanwhile, a PacBio RS II library was constructed for both isolates and sequenced using P4-C2 chemistry with 16 SMRT cells, generating 5.55 and $6.27 \mathrm{~Gb}$ reads for Pst (93-210) and Psh (93TX-2), respectively. Trimmomatic (version 0.36) [60], Lighter [61] and Proovread v2.14.0 [62] were used to correct the potential sequencing errors in the Illumina and PacBio long reads. Genome assembly and annotation were outlined in Additional file 1: Figure S8. More detailed steps for assembly, polishing, and annotation can be found in our related paper [63].

One additional Psh isolate (04-051) was sequenced using the Ion Proton technology following the previously described method [64]. This isolate was collected from Oregon, Washington in 2004 and was virulent on barley differentials Topper, Heils Franken, Hiproly, Abed Binder 12, Trumpf, I 5 and Bancroft but avirulent on the remaining barley differentials (Emir, Astrix, Varunda,
Mazurka and Bigo) and all wheat differentials for identifying Pst races. The sequences of this isolate was used to determine whether the genes specifically present or absent in Psh (93TX-2) were also the same in this isolate.

\section{K-mer analysis and genome size estimation}

Program Jellyfish version 2.2.6 [65] was used to analyze the distribution of $K$-mer and to estimate the genome size using only filtered and corrected Illumina reads. According to the Lander-Waterman theory [66], the genome size can be estimated using the total number of $K$-mers divided by the peak value in the $K$-mer distribution. We found that both Pst and Psh isolates were heterozygous since both commonly had two peaks in the $K$-mer distribution (Additional file 1: Figure S1). Then the genome size was calculated separately for regions with and without heterozygosity. With the peak at the expected $K$-mer depth, the calculated genome size, which equals the total $K$-mer/expected $K$-mer depth, was estimated for each region.

Two peaks of 17-mer were observed at the depth of 19 and 33 for isolate Pst (93-210), representing two distinct heterozygous and homozygous genomic regions, respectively. The total estimated genome size was $89,743,503 \mathrm{bp}$, of which 20,535,944 bp and 69,207,559 bp were estimated to be in the heterozygous and homozygous regions, respectively. Similarly, two peaks at 15 and 26 were observed for isolate Psh (93TX-2). The estimated genome size was $89,709,453 \mathrm{bp}$, of which $18,880,342 \mathrm{bp}$ and $70,829,111 \mathrm{bp}$ were estimated to be in the heterozygous and homozygous regions, respectively. 


\section{Analysis of telomeres}

The gapless haploid genomes in this study enabled us to accurately survey the telomere regions that might be often unassembled in previously available cereal rust genomes. Telomere repeats were identified by manually searching the ends of contigs for short, high-copy-number (>30) repeats.

\section{Transcriptome sequencing}

RNA sequencing was conducted for both Pst (93-210) and Psh (93TX-2). Total RNA was extracted from fresh urediniospores, germinated urediniospores and infected wheat leaves 2 and 7 days after inoculation. Fresh urediniospores, germinated urediniospores and infected leaves were grounded separately in a mortar in liquid nitrogen. Total RNA was extracted from frozen powder using the Trizol reagent (Thermo Fisher Scientific, MA) following the manufacturer's instruction. RNA quality was checked using the Agilent ${ }^{\circ} 2100$ Bioanalyzer $^{\circ}$ (Sgilent Technologies, UK). The RNA samples from different stages of each isolate were mixed into one sample at the 1:1:1:1 ratio for RNA sequencing. The preparation of RNA-Seq library and Illumina HiSeq PE150 sequencing were performed at the Novogen Corporation Inc. (Sacramento, CA). Raw reads of RNA-Seq were checked and trimmed using the same methods as described above. In total, 19,802,677 and 25,432,126 paired-end reads of Pst and Psh were obtained and used for subsequent analyses. The filtered RNA-Seq reads were used for both genome annotation and gene expression estimation.

\section{SNP and InDel analyses}

A previously proposed framework for discovering variation was followed [67]. To identify intra-isolate SNPs and InDels, the high quality Illumina reads of each isolate were mapped to its respective assembled genome as a reference, and high quality SNPs and InDels were called from the alignments. The reference genome was indexed using the Burrows-Wheeler Alignment (BWA) Tool version 0.7.15 [68]. High quality paired-end Illumina reads were mapped to the reference using the mem algorithm with the default parameters except $-r$ 1.0. The SAM formatted alignment was converted to the BAM format after removing the duplicates using SAMtools version 1.2 [69]. The BAM files were cleaned, validated and sorted using the Picard Tools version 1.129 (http://picard.sourceforge.net/), and then indexed using SAMtools. To reduce the number of potentially mismatching Indels in the alignment, the local realignment was conducted following two steps using the Genome Analysis Toolkit (GATK) version 3.3 [67]. First, the interval targets for local realignment were defined using GATK's RealignerTargetCreator with the default parameters. Second, GATK's IndelRealigner was used to perform indel realignment of reads around defined targets from the previous step.

To improve the accuracy of base qualities for subsequent variant calling, we performed a Base Quality Score Recalibration step to detect systematic errors made during the sequencing when estimating the quality score of each base call. Firstly, we performed an initial round of variant calling using GATK HaplotypeCaller. SNPs and InDels of high confidence were filtered based on the Quality value in vcf using vcftools (version 0.1.13) with parameters set as --min-alleles 2 --max-alleles 2 --minQ 1000 (meaning that only biallelic SNPs and InDels with a minimum Quality of 1000 were kept). Then the filtered high confidence SNPs and InDels were used as known variants to model systemic sequencing errors empirically, and the base qualities were adjusted using GATK BaseRecalibrator. Lastly, the recalibrated BAM was generated using GATK PrintReads. The second round of variant calling was performed using GATK HaplotypeCaller. To finalize, the high quality SNPs and InDels with minimum calling quality of 100 were selected. Furthermore, we only kept SNPs and InDels within coverages 20-120X to reduced false positives in repetitive regions where Illumina reads from multiple different copies of the repeat were mapped to a single locus. These were done using vcftools with the parameters set as --min-meanDP 20 --max-meanDP 120 -minQ 100.

The variations (SNPs and InDels) were annotated and their effects were predicted using software SnpEff version 4.3r [70]. Firstly, the databases of Pst and Psh were built locally using annotations in the GTF format generated from the genome annotation section (described above). The SnpEff databases were subsequently used to annotate intra- and inter-isolate SNPs and InDels with putative functional effects according to categories defined in the SnpEff $\mathrm{v} 4.3 \mathrm{r}$ manual. Other parameters were set as default, e.g. the upstream and downstream interval sizes of $5000 \mathrm{bp}$. Predicted effects are given in Additional file 3: Table S4. As defined by SnpEff, some of the high impacts include exon_loss, frameshift, splice_site_acceptor, splice_site_donor, start_loss, stop_lost and stop_gained. It should be noted that SnpEff allows variations to be included in multiple categories, so the total number of effects does not exactly match the total number of variants in Additional file 3: Table S4.

\section{Synteny analysis}

Whole genome comparison between Pst (93-210) and Psh (93TX-2) was conducted to evaluate gene synteny using the MUMmer 3.23 package [71]. Specifically, the alignments of nucleotide contigs were generated from NUCmer using the option --maxmatch set to include all match sequences regardless of uniqueness and the 
defaults for other parameters. The delta encoded alignments were filtered using delta-filter by setting -i $90-1$ 1000 -r -q -o 10 to keep only the alignments with identities above 90\% and length above $1000 \mathrm{bp}$. For visualization, plots were generated using mummerplot and Gnuplot, visualized using program circos version 0.69-5 [72]. The total size of syntenic regions was calculated by adding length of all alignments, and the overall identity was calculated by averaging the identities of all aligned blocks. Also compared were Pst (93-210) versus Pst (104E 137A-) and Psh (93TX-2) versus Pst (104E 137A-) [23].

\section{Ortholog detection and analysis}

Protein orthologous groups (orthogroups) were inferred using OrthoFinder version 1.1.8 [73] with the default parameters. Protein sequences of 16 plant fungal pathogens including 4 Ascomycetes and 12 Basidiomycetes were retrieved from the NCBI or JGI database (Additional file 2: Table SE9). To infer orthogroups, an all-vs-all BLAST search was firstly performed, and then the resulting e-values were used for clustering using the MCL program.

The isolate-specific genes, 861 in Pst (93-210) and 955 in Psh (93TX-2), were searched against the NCBI non-redundant protein database (updated on March 6, 2017; downloaded September 25, 2017) using blastp v2.4.0 to identify their potential origin, e.g. via horizontal gene transfer (HGT). To identify their genomic features, we compared the isolate-specific genes in Pst (93-210) and Psh (93TX-2) with 452 single-copy protein-coding genes identified from 16 fungi using OrthoFinder. The reason behind this is that the isolate-specific genes represent a group of rapidly evolving genes in these isolates, whereas the 452 single-copy protein-coding genes are present in all 16 fungi and therefore are highly conserved. We first calculated the lengths of isolate-specific genes in Pst (93-210) and Psh (93TX-2). Secondly, we mapped our RNA-Seq reads to the respective reference genomes to estimate the gene expression level. Then the gene expression level was quantified from the gene abundance file generated using program StringTie v1.3.3b [74] and normalized in the number of fragments per kilobase of transcript per million read pairs (FPKM). Thirdly, we calculated the genomic distances of isolate-specific genes to the closest transposable elements (TEs) at both $5^{\prime}$ and $3^{\prime}$ ends, and compared the values to those of the conserved genes. These features were visualized using the 'beanplot' [75] (Fig. 5a-d) and 'ggplot2' [76] (Fig. 5e-h) packages in program R.

To test the hypothesis that the rapidly evolving isolate-specific genes may be subject to loss-of-function, the isolate-specific genes were searched against the nonredundant set of KEGG (Kyoto Encyclopedia of Genes and Genomes) genes using software GhostKOALA version 2.0 [77].

Statistical analysis of the GO-term enrichment was conducted using program Fatigo in Blbelomics 5 [78]. Specifically, we compared the GO terms: 1) of all proteins of Pst (93-210) and Psh (93TX-2); 2) of proteins involved in loss events with the rest of the proteins in Pst (93-210) and Psh (93TX-2); and 3) of isolate-specific proteins with the rest of the proteins in Pst (93-210) and Psh (93TX-2). No significant GO term enrichment was detected in all comparisons.

\section{Divergence time and mutation rate}

The proteins from 452 single-copy orthogroups were used to infer the phylogenomic relationship and divergence time of Pst and Psh. Peptide alignment was conducted using CLUSTAL Omega version 1.2.4 [79]. Only sites without gaps in the alignment and alignments with at least 50 amino acids in length were kept in the subsequent analyses, resulting in 158,428 amino acid sites and 439 proteins. Phylogenomics inference was conducted using the Bayesian-based method in program MrBayes v3.2.6 [80], following the procedure described previously [64]. A partitioned analysis was set up for MrBayes so that different models could be estimated and applied for each protein. For each protein, the evolutionary model was selected based on ProtTest 3.4.2 [81]. The divergence times of Pst and Psh and of other fungal plant pathogens used in this study were estimated using a semiparametric Penalized Likelihood (PL) method with the Truncated-Newton (TN) algorithm in r8s version 1.81 [82]. Cross-validation was used to explore the fidelity of the method to explain the branch length variation. To do this, we tried to optimize a range of smoothing parameters, at increments of 1.0, from -8 to 2 on a $\log _{10}$ scale. For our dataset, the smoothing value of 1e-05 gave the lowest cross-validation score, and therefore was used to obtain the optimal estimates of the divergence time and rate. To do such estimation, at least one calibration time is needed. We calibrated the times on the tree based on the divergence between Ascomycota and Basidiomycota at the minimum age of 452 million years that was previously estimated when a fossil of Paleopyrenomycites was considered to be in Ascomycota [83]. Another reason we used this divergence time was that this calibration time generated very close divergence for Blumeria graminis f. sp. tritici and B. graminis f. sp. hordei compared to a previous estimation on the basis of substitutions in synonymous sites [9.22 myrs versus $6.3( \pm 1.1)$ myrs] [24]. To further validate our estimated divergence time between Pst and Psh, we calculated the substitution rate between these two genomes. We selected 5611 pairs of single-copy protein homologs of the Pst and Psh isolates (see the $\mathrm{dN} / \mathrm{dS}$ 
analysis section) and identified 1,307,578 bp substitutions from a total length of 8,025,786 bp for these genes. Based on these data, we calculated the mutation rate as $2.0 \times 10^{-8}$ per base per year, which is within a previous reported Pst mutation rate range of $1.7 \times 10^{-7}$ to $8.6 \times$ $10^{-8}$ per base pair per generation based on 17,000 AFLP fragments [84].

\section{$\mathrm{dN} / \mathrm{dS}$ analysis}

The method of Yang and Nielsen [85] was implemented in program yn00 of the PAML package version 4.9e [86], and the transition/transversion rate bias and codon usage bias were counted for estimating the $\mathrm{dN} / \mathrm{dS}$ ratios, following the previously described method [24]. Firstly, the 5611 pairs of single-copy protein homologs of Pst and Psh were determined using OrthoFinder and aligned using CLUSTAL Omega. Then the protein alignment was used as an anchor to generate the corresponding CDS alignment to assure the corresponding bases were aligned, which is an essential requirement for $\mathrm{dN} / \mathrm{dS}$ analyses. This step was achieved using program PAL2NAL [87], which converts protein alignment and the corresponding DNA sequences into a codon-based DNA alignment. The outputs in the paml format were concatenated and subjected to the yn00 analysis for $\mathrm{dN} /$ $\mathrm{dS}$ estimation. The $\mathrm{dN} / \mathrm{dS}$ ratios were assessed separately for the SP-coding genes and non-SP-coding genes to test if the two groups had different characteristics of natural selection. It should be noted that it is impossible to calculate $\mathrm{dN} / \mathrm{dS}$ when there are nonsynonymous substitutions but no synonymous substitutions in a gene sequence $(d N \neq 0$, while $d S=0)$. In these situations, we arbitrarily designated $\mathrm{dN} / \mathrm{dS}=100$, and plotted as $>3$ in Fig. 3b.

\section{Evolution analysis of protein-coding gene families}

Protein-coding gene families of 16 plant fungal pathogens were assigned using OrthoFinder as described above. The evolution of protein family loss and gain was analyzed following the method described previously [41]. First, the presence and absence information of each family in each species was prepared manually and stored in the Phlylip format. Then the DOLLOP program from the PHYLIP version 3.696 [88] was used to reconstruct a phylogenic tree using the Dollo parsimony method, and also to estimate the protein family loss and gain events for each species and for internal nodes of the phylogenetic tree.

\section{Identification of isolate-specifically expressed genes}

To identify genes exclusively expressed in either Pst (93-210) or Psh (93TX-2), the filtered RNA-Seq reads generated from both isolates were mapped to Pst and Psh separately using program HISAT2 v2.1.0. The alignments were converted and sorted in the BAM format. Then gene expression level was quantified from the gene abundance file generated using program StringTie v1.3.3b [74]. A gene was considered as form-exclusively expressed when the normalized expression level in FPKM was $>1.0$ using the RNA-Seq reads from Pst or Psh but the FPKM was 0 using reads of the other isolate.

\section{Analysis of specific protein-coding gene families}

Putative SPs were predicted following a previously recommended pipeline $[89,90]$. Briefly, the presence and location of signal peptide cleavage sites were predicated using SignalP version 3.0. The presence of secretory pathway signal peptide was confirmed using TargetP version 1.1. The proteins with predicted chloroplast transit peptides and mitochondrial targeting peptides were removed. The presence of transmembrane helices was predicted using TMHMM version 2.0 (http://www.cbs.dtu.dk/services/TMHMM/). Finally, the proteins with the presence of signal peptides and absence of neither chloroplast and mitochondrial targets transmembrane helices nor transmembrane helices were filtered for subsequent analyses.

To test whether specific carbohydrate-active enzymes were associated with host adaptation, we performed CAZyme annotation for two wheat-hosted fungi (Pst and $B g t)$ and two barley-hosted fungi (Pgh and Bgh). Predicted proteins of four fungi were searched against entries in the CAZy database (http://www.cazy.org) using the dbCAN Web server, an automated carbohydrate-active enzyme annotation pipeline based on the CAZyme family-specific domains [91].

Secondary metabolite (SM) anchor genes were predicted using program SMIPS [92] based on protein domain annotations using InterProScan as described above.

As membrane transporters, which have functions to transfer nutrients, metabolism products, signaling molecules and many other cellular constituents from source to sink for cellular uptake, are of particular importance for obligate biotrophic fungi, we searched membrane transporter genes. To do this, TCDB (Transporter Classification Database) sequences [93] in the fasta format were retrieved from http://www.tcdb.org/download.php (October 16th, 2017). The TCDB sequences were searched using Blastp (percentage identity $>30 \%$; e-value $<1 \mathrm{e}-50)$ to predict transporters in Pst $(93-210)$, Psh (93TX-2), Bgt and Bgh.

Transcription factors were predicted based on the assignment of proteins to specific DNA-binding domain families using the Pfam libraries. The domain assignments were performed on complete genome proteins using InterProScan. Transcription factor families were 
classified as described in the transcription factor prediction database (http://www.transcriptionfactor.org).

Peptidases (also termed proteases, proteinases and proteolytic enzymes) and inhibitors were predicted in the four fungal (Pst, Psh, Bgt and Bgh) genomes using the MEROPS database (release 12.0) [94] which uses a hierarchical, structure-based classification of the peptidases. Proteins of the four fungi were searched against the MEROPS database, which was retrieved from $\mathrm{ftp}: / /$

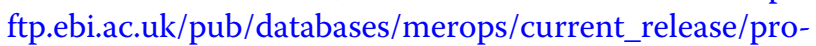
tease.lib (October 24th, 2017). The peptidase or inhibitor family of the best hit (percentage identity $>30 \%$; e-value $<1 \mathrm{e}-50)$ from the blastp results was assigned to the query protein.

\section{Coverage analysis and detection of genomic region deletions}

Two Pst isolates (93-210 and 2K-41-Yr9) and two Psh isolates (93TX-2 and 04-051) were used to detect forma specialis-specific regions. The genomic sequence of 2K-41-Yr9 (PST-78) was from http://genome.jgi.doe.gov/ pages/dynamicOrganismDownload.jsf?organism $=$ Pucst PST78_1 (Additional file 2: Table SE9), and the genomic sequence of Psh 04-51 was obtained in this study as mentioned above. We defined the genomic regions as Pst-specific regions when: 1) these regions were covered by mapping sequence reads of Pst isolates to the Pst reference genome; and 2) these regions were not covered by mapping sequence reads of Psh isolates to the Pst reference genome. Similarly, we also defined $P$ sh-specific regions. The sequence reads of all four isolates were mapped to the Pst (93-210) and Psh (93TX-2) references using the method described above. We calculated the mapping coverage based on the recalibrated alignments in the BAM format using the bedcov module in SAMtools with a window size of $500 \mathrm{bp}$. The region was considered as deleted in the mapping isolate when the mapping coverage was 0 . Selected deletion regions were visualized using Circos (Additional file 1: Figure S4).

\section{Additional files}

Additional file 1: Figure S1. The 17-mer depth distributions of the Illumina reads used to estimate the genome sizes of Pst (93-210) and Psh (93TX-2) isolates of Puccinia striiformis used in this study. Figure S2. Comparisons of functional annotations between wheat-hosted and barley-hosted stripe rust and powdery mildew fungi. Figure S3. Collinearity analysis of syntenic blocks among three Puccinia striiformis isolates. Figure S4. Examples of missing genomic regions by reciprocal mapping of Illumina sequence reads. Figure S5. An overview of a large syntenic region between isolates PS (93-210) and Psh (93TX-2) of Puccinia striiformis. 4. Figure S6. An overview of the mitochondrial $(\mathrm{mt})$ genomes of isolates Pst (93-210) and Psh (93Tx-2) representing Puccinia striiformis f. sp. tritici (Pst) and P. striiformis f. sp. hordei (Psh), respectively. Figure S7. IGV screenshot showing examples of exclusively expressed genes revealed by reciprocal mapping of Illumina RNA-Seq reads. Figure S8. Genome assembly pipeline used for genome assembly in this study. See details in Methods. (DOCX $4535 \mathrm{~kb}$ )

Additional file 2: Table SE1. Summary of transposable elements in Puccinia striiformis f. sp. tritici and P. striiformis f. sp. hordei genomes Table SE2. Comparison of the annotated CAZyme genes among Puccinia striiformis f. sp. tritici (Pst), P. striiformis f. sp. hordei (Psh), Blumeria graminis f. sp. tritici (Bgt) and B. graminis f. sp. hordei (Bgh). Table SE3. Matingrelated genes in Puccinia striiformis f. sp. tritici (Pst), P. striiformis f. sp. hordei (Psh), Blumeria graminis f. sp. tritici (Bgt) and B. graminis f. sp. hordei (Bgh). Table SE4. Comparison of the annotated peptidases genes among Puccinia striiformis f. sp. tritici (Pst), P. striiformis f. sp. hordei (Psh), Blumeria graminis f. sp. tritici (Bgt) and B. graminis f. sp. hordei (Bgh). Table SE5. Comparison of the annotated genes in isolate Pst (93-210) (Table SE5a) and Psh (93TX-2) (Table SE5b) using the Pathogen-Host-Interaction database. Table SE6. Summary of secondary-metabolite gene classes in 17 fungal plant pathogens. Table SE7. Comparison of the annotated transcription factor genes among nine fungal species or formae speciales. Table SE8. Comparison of the annotated transporter genes among Puccinia striiformis f. sp. hordei (Psh); P. striiformis f. sp. tritici (Psh); Blumeria graminis f. sp. hordei (Bgh) and B. graminis f. sp. tritici (Bgt). Table SE9. The list of fungal genomes and their resources used in this study. Table SE10. Summary of orthologues detected in 16 fungal plant pathogens using the OrthoFinder program. Table SE11. KEGG pathways annotated using GhostKOALA for Puccinia striiformis f. sp. tritici (Pst) and P. striiformis f. sp. hordei (Psh) isolates. Table SE11a. The numbers of conserved, specific and lost genes in Pst (93-210) and Psh (93TX-2); Table SE11b. Annotation of genes of KEGG pathways in Pst (93-210); and Table SE11c. Annotation of genes of KEGG pathways in Psh (93TX-2). Table SE12. List of exclusively expressed isolate-specific genes in isolates Pst (93-210) and Psh (93Tx-2) of Puccinia striiformis absent in the genome of the opposite isolate. Table SE13. List of exclusively expressed genes in isolates Pst (93-210) and Psh (93Tx-2) of Puccinia striiformis that are present in the genomes of both isolates. (ZIP $1140 \mathrm{~kb}$ )

Additional file 3: Table S1. The assessment of genome annotations of Puccinia striiformis f. sp. tritici (Pst) and P. striiformis f. sp. hordei (Psh) using the BUSCO program [95]. Table S2. Annotated conserved protein-coding and rRNA genes in the mitochondrial genomes of Puccinia striiformis $\mathrm{f}$. sp. tritici [Pst (93-210)] and P. striiformis f. sp. hordei [Psh (93TX-2)]. Table S3. Transfer RNA genes in the mitochondria genomes of Puccinia striiformis $f$. sp. tritici [Pst (93-210)] and P. striiformis f. sp. hordei [Psh (93TX-2)].

Table S4. Predicted numbers of variant effects by type in comparison of Puccinia striiformis f. sp. tritici (Pst) and P. striiformis f. sp. hordei (Psh). Table S5. Summary functions of genes in isolates Pst (93-210) and Psh (93TX-2) of Puccinia striiformis impacted by mutations and under positive selective. (DOCX 45 kb)

\section{Abbreviations}

Avr: Avirulence gene; GO: Gene Ontology; HR: Hyposensitive Reaction; KEGG: Kyoto Encyclopedia of Genes and Genomes; MRCA: Most Recent Common Ancestor; mt: mitochondria; Psh: Puccinia striiformis f. sp. hordei; Pst: Puccinia striiformis f. sp. tritici; SP: Secreted Protein; TE: transposable element; TF: Transcription Factor

\section{Acknowledgements}

This study was conducted in the facilities of U.S. Department of Agriculture, Agricultural Research Service and Washington State University (WSU), Pullman, Washington. Department of Plant Pathology, College of Agricultural, Human, and Natural Resource Sciences, Agricultural Research Center, Project No. WNP00663, Washington State University, Pullman, WA 99164-6430, USA. Technical support from WSU Information Technology staff is highly appreciated. The authors would like to thank Drs. Tobin Peever and Weidong Chen from WSU and Prof. Huiquan Liu from Northwest A\&F University, Yangling, China for critical review of the manuscript. The China Scholarship Council scholarship to CX is highly appreciated. We also acknowledge the Kamiak high performance computing clusters in WSU for providing computing resources. 
recommendation or endorsement by the U. S. Department of Agriculture. USDA is an equal opportunity provider and employer.

\section{Funding}

This study was supported by the U.S. Department of Agriculture, Agricultural Research Service (Project No. 2090-22000-018-00D) and Washington Grain Commission (Projects 13C-3061-5682).

\section{Availability of data and materials}

The data of this study data have been deposited in the NCBI with BioProject accession PRJNA422914, sample accessions SAMN08200485 for Pst (93-210) and SAMN0800486 for Psh (93TX-2). Genome assembly and annotation have been deposited in the NCBI with accessions PKSL00000000 for Pst (93-210) and PKSM00000000 for Psh (93TX-2).

\section{Authors' contributions}

CX, MW, and CY participated in the laboratory experiments. CX participated in producing spores, and extracting genomic DNA and RNA; MW participated in selecting isolates, inoculating plants, and extracting genomic DNA; CY participated in extracting and quantifying RNA. CX, OC, SH, and XC analyzed, interpreted the data, and wrote manuscript. CX performed most bioinformatic analyses, and a major contributor in writing the manuscript; OC guided data analyses, interpreted data and revising the manuscript; $\mathrm{SH}$ guided the study, contributed resources, interpreted data and revised the manuscript; XC conceived and coordinated the study, designed the experiments, provided materials and resources, interpreted data and wrote the manuscript. All authors read and approved the final manuscript.

\section{Ethics approval and consent to participate}

Not applicable.

\section{Consent for publication}

Not applicable.

\section{Competing interests}

The authors declare that they have no competing interests.

\section{Publisher's note}

Springer Nature remains neutral with regard to jurisdictional claims in published maps and institutional affiliations.

\section{Author details}

'Department of Plant Pathology, Washington State University, Pullman, WA 99164-6430, USA. ${ }^{2}$ School of Biological Sciences, Washington State University, Pullman, WA 99164-7520, USA. ${ }^{3}$ Wheat Health, Genetics, and Quality Research Unit, Agriculture Research Service, U.S. Department of Agriculture, Pullman, WA 99164-6430, USA.

Received: 6 May 2018 Accepted: 27 August 2018 Published online: 12 September 2018

\section{References}

1. Fisher MC, Henk DA, Briggs CJ, Brownstein JS, Madoff LC, MCCraw SL, et al. Emerging fungal threats to animal, plant and ecosystem health. Nature. 2012:484:186-94.

2. Stukenbrock EH, McDonald BA. The origins of plant pathogens in agroecosystems. Annu Rev Phytopathol. 2008;46:75-100.

3. Dong S, Raffaele S, Kamoun S. The two-speed genomes of filamentous pathogens: waltz with plants. Curr Opin Genet Dev. 2015;35:57-65.

4. Möller M, Stukenbrock EH. Evolution and genome architecture in fungal plant pathogens. Nature Rev Microbiol. 2017;15:756-71.

5. Raffaele S, Kamoun S. Genome evolution in filamentous plant pathogens: why bigger can be better. Nature Rev Microbiol. 2012;10:417-30.

6. Kämper J, Kahmann R, Bölker M, Ma L-J, Brefort T, Saville BJ, et al. Insights from the genome of the biotrophic fungal plant pathogen Ustilago maydis. Nature. 2006:444:97-101.

7. Haas BJ, Kamoun S, Zody MC, Jiang RH, Handsaker RE, Cano LM, et al. Genome sequence and analysis of the Irish potato famine pathogen Phytophthora infestans. Nature. 2009;461:393-8.

8. Rouxel T, Grandaubert J, Hane JK, Hoede C, van de Wouw AP, Couloux A, et al. Effector diversification within compartments of the Leptosphaeria maculans genome affected by repeat-induced point mutations. Nat Commun. 2011;2:202.

9. Coleman JJ, Rounsley SD, Rodriguez-Carres M, Kuo A, Wasmann CC, Grimwood J, et al. The genome of Nectria haematococca: contribution of supernumerary chromosomes to gene expansion. PLoS Genet. 2009;5: e1000618.

10. Ma L-J, van Der Does HC, Borkovich KA, Coleman JJ, Daboussi M-J, Di Pietro A, et al. Comparative genomics reveals mobile pathogenicity chromosomes in fusarium. Nature. 2010;464:367-73.

11. Chen $X$, Introduction KZ. History of research, symptoms, taxonomy of the pathogen, host range, distribution, and impact of stripe rust. In: Chen $X$, Kang Z, editors. Stripe Rust: Springer; 2017. p. 1-33.

12. Cheng $P$, Chen $X$, See $D$. Grass hosts harbor more diverse isolates of Puccinia striiformis than cereal crops. Phytopathology. 2016;106:362-71.

13. Chen $X$, Line RF, Leung $H$. Virulence and polymorphic DNA relationships of Puccinia striiformis f. Sp. hordei to other rusts. Phytopathology. 1995:85:1335-42.

14. Troch V, Audenaert K, Wyand RA, Haesaert G, Höfte M, Brown JK. Formae speciales of cereal powdery mildew: close or distant relatives? Mol Plant Pathol. 2014;15:304-14.

15. Gordon TR. Fusarium oxysporum and the fusarium wilt syndrome. Annu Rev Phytopathol. 2017;55:23-39.

16. Poloni A, Schirawski J. Host specificity in Sporisorium reilianum is determined by distinct mechanisms in maize and sorghum. Mol Plant Pathol. 2016;17:741-54

17. Menardo F, Praz CR, Wyder S, Ben-David R, Bourras S, Matsumae H, et al. Hybridization of powdery mildew strains gives rise to pathogens on novel agricultural crop species. Nature Genet. 2016;48:201-5.

18. Cantu D, Govindarajulu M, Kozik A, Wang M, Chen X, Kojima KK, et al. Next generation sequencing provides rapid access to the genome of Puccinia striiformis f. Sp. tritici, the causal agent of wheat stripe rust. PLoS One. 2011;6:e24230

19. Cantu D, Segovia V, MacLean D, Bayles R, Chen X, Kamoun S, et al. Genome analyses of the wheat yellow (stripe) rust pathogen Puccinia striiformis $\mathrm{f}$. Sp. tritici reveal polymorphic and haustorial expressed secreted proteins as candidate effectors. BMC Genomics. 2013;14:270.

20. Cuomo CA, Bakkeren G, Khalil HB, Panwar V, Joly D, Linning R, et al. Comparative analysis highlights variable genome content of wheat rusts and divergence of the mating loci. G3 (Bethesda). 2017;7:371-6.

21. Kiran K, Rawal HC, Dubey H, Jaswal R, Bhardwaj SC, Prasad P, et al. Dissection of genomic features and variations of three pathotypes of Puccinia strifformis through whole genome sequencing. Sci Rep. 2017;7:42419.

22. Zheng W, Huang L, Huang J, Wang X, Chen X, Zhao J, et al. High genome heterozygosity and endemic genetic recombination in the wheat stripe rust fungus. Nat Commun. 2013:4:2673.

23. Schwessinger B, Sperschneider J, Cuddy W, Miller M, Garnica D, Taylor J, et al. A near complete haplotype-phased genome of the dikaryotic wheat stripe rust fungus Puccinia striiformis $\mathrm{f} p$ tritici reveals high interhaplotype diversity. mBio. 2018:9:e02275-17.

24. Wicker T, Oberhaensli S, Parlange F, Buchmann JP, Shatalina M, Roffler S, et al. The wheat powdery mildew genome shows the unique evolution of an obligate biotroph. Nature Genet. 2013;45:1092-6.

25. Spanu PD, Abbott JC, Amselem J, Burgis TA, Soanes DM, Stüber K, et al. Genome expansion and gene loss in powdery mildew fungi reveal tradeoffs in extreme parasitism. Science. 2010:330:1543-6.

26. Akhunov ED, Goodyear AW, Geng S, Qi L-L, Echalier B, Gill BS, et al. The organization and rate of evolution of wheat genomes are correlated with recombination rates along chromosome arms. Genome Res. 2003;13:753-63.

27. Chalupska D, Lee H, Faris J, Evrard A, Chalhoub B, Haselkorn R, et al. Acc homoeoloci and the evolution of wheat genomes. Proc Natl Acad Sci. 2008;105:9691-6.

28. Langergraber KE, Prüfer K, Rowney C, Boesch C, Crockford C, Fawcett K, et al. Generation times in wild chimpanzees and gorillas suggest earlier divergence times in great ape and human evolution. Proc Natl Acad of Sci. 2012;109:15716-21.

29. Xia C, Wan A, Wang M, Jiwan DA, See DR, Chen X. Secreted protein gene derived-single nucleotide polymorphisms (SP-SNPS) reveal population diversity and differentiation of Puccinia striiformis f. Sp. tritici in the United States. Fungal Biol. 2016;120:729-44.

30. Liu B, Chen X, Kang Z. Gene sequencing reveals heterokaryotic variations and evolutionary mechanisms in Puccinia striiformis. Open J Genomics. $2012 ; 1: 1$. 
31. Chen J, Upadhyaya NM, Ortiz D, Sperschneider J, Li F, Bouton C, et al. Loss of AvrSr50 by somatic exchange in stem rust leads to virulence for Sr50 resistance in wheat. Science. 2017;358:1607-10.

32. Berlin A, Djurle A, Samils B, Yuen J. Genetic variation in Puccinia graminis collected from oats, rye, and barberry. Phytopathology. 2012;102:1006-12.

33. Cheng $P$, Chen $X$. Virulence and molecular analyses support asexual reproduction of Puccinia striiformis $\mathrm{f}$. Sp. tritici in the US Pacific northwest. Phytopathology. 2014;104:1208-20.

34. Wang $M$, Chen X. Barberry does not function as an alternate host for Puccinia striiformis f. Sp. tritici in the US Pacific northwest due to teliospore degradation and barberry phenology. Plant Dis. 2015;99:1500-6.

35. Wang M, Wan A, Chen X. Barberry as alternate host is important for Puccinia graminis f. Sp. tritici but not for Puccinia striiformis f. Sp. tritici in the US Pacific northwest. Plant Dis. 2015;99:1507-16.

36. Aime MC, McTaggart AR, Mondo SJ, Duplessis S. Phylogenetics and phylogenomics of rust fungi. Advan Genet. 2017;100:267-307.

37. Tautz D, Domazet-Lošo T. The evolutionary origin of orphan genes. Nature Rev Genet. 2011;12:692-702.

38. Petre B, Joly DL, Duplessis S. Effector proteins of rust fungi. Front Plant Sci. 2014,5:416.

39. van Dam P, Fokkens L, Schmidt SM, Linmans JH, Kistler HC, Ma LJ, et al. Effector profiles distinguish formae speciales of Fusarium oxysporum. Environ Microbiol. 2016:18:4087-102.

40. Albalat R, Cañestro C. Evolution by gene loss. Nature Rev Genet. 2016;17: 379-91.

41. Duplessis S, Cuomo CA, Lin Y-C, Aerts A, Tisserant E, Veneault-Fourrey C, et al. Obligate biotrophy features unraveled by the genomic analysis of rust fungi. Proc Natl Acad Sci. 2011;108:9166-71.

42. Gout L, Kuhn ML, Vincenot L, Bernard-Samain S, Cattolico L, Barbetti M, et al. Genome structure impacts molecular evolution at the AvrLm1 avirulence locus of the plant pathogen Leptosphaeria maculans. Environm Microbiol. 2007:9:2978-92.

43. Chuma I, Isobe C, Hotta Y, Ibaragi K, Futamata N, Kusaba M, et al. Multiple translocation of the AVR-Pita effector gene among chromosomes of the rice blast fungus Magnaporthe oryzae and related species. PLoS Pathog. 2011;7:e1002147.

44. Schürch S, Linde CC, Knogge W, Jackson LF, McDonald BA. Molecular population genetic analysis differentiates two virulence mechanisms of the fungal avirulence gene NIP1. Mol Plant-Microbe Interact. 2004;17:1114-25.

45. Salcedo A, Rutter W, Wang S, Akhunova A, Bolus S, Chao S, et al. Variation in the AvrSr35 gene determines Sr35 resistance against wheat stem rust race Ug99. Science. 2017;358:1604-6.

46. Pahalawatta $V$, Chen $X$. Inheritance and molecular mapping of barley genes conferring resistance to wheat stripe rust. Phytopathology. 2005;95:884-9.

47. Wan A, Chen X. Virulence, frequency, and distribution of races of Puccinia striiformis f. Sp. tritici and P. striiformis f. Sp. hordei identified in the United States in 2008 and 2009. Plant Dis. 2012;96:67-74.

48. Liu T, Wan A, Liu D, Chen X. Changes of races and virulence genes in Puccinia striiformis $\mathrm{f}$. Sp. tritici, the wheat stripe rust pathogen, in the United States from 1968 to 2009. Plant Dis. 2017;101:1522-32.

49. Wan A, Chen X, Yuen J. Races of Puccinia striiformis f. Sp. tritici in the United States in 2011 and 2012 and comparison with races in 2010. Plant Dis. 2016;100:966-75.

50. Meyne J, Ratliff RL, Moyzis RK. Conservation of the human telomere sequence (TTAGGG)n among vertebrates. Proc Natl Acad Sci U S A. 1989:86:7049-53.

51. Wu C, Kim YS, Smith KM, Li W, Hood HM, Staben C, et al. Characterization of chromosome ends in the filamentous fungus Neurospora crassa. Genetics. 2009;181:1129-45

52. Goddard MV. Cytological studies of Puccinia striiformis (yellow rust of wheat). Trans Br Mycol Soc. 1976;66:433-7.

53. Yuan CY, Wang MN, Skinner DZ, See DR, Xia CJ, Guo XH, et al. Inheritance of virulence, construction of a linkage map, and mapping of virulence genes in Puccinia striiformis f. Sp. tritici by virulence and molecular characterization of a sexual population through genotyping-by-sequencing. Phytopathology. 2018;108:133-41

54. Eisenberg DTA. An evolutionary review of human telomere biology: the thrifty telomere hypothesis and notes on potential adaptive paternal effects. Am J Human Biol. 2011;23:149-67.

55. McTaggart AR, Shivas RG, Nest MA, Roux J, Wingfield BD, Wingfield MJ. Host jumps shaped the diversity of extant rust fungi (Pucciniales). New Phytol. 2016;209:1149-58
56. Lei $Y$, Wang M, Wan A, Xia C, See DR, Zhang M, et al. Virulence and molecular characterization of experimental isolates of the stripe rust pathogen (Puccinia striiformis) indicate somatic recombination. Phytopathology. 2016;107:329-44.

57. Hovmøller MS, Sørensen CK, Walter S, Justesen AF. Diversity of Puccinia striiformis on cereals and grasses. Annu Rev Phytopathol. 2011:49:197-217.

58. Wan A, Chen X. Virulence characterization of Puccinia striiformis f. Sp. tritici using a new set of $Y r$ single-gene line differentials in the United States in 2010. Plant Dis. 2014:98:1534-42.

59. Murray MG, Thompson WF. Rapid isolation of high molecular weight plant DNA. Nucleic Acids Res. 1980:8:4321-6.

60. Bolger AM, Lohse M, Usadel B. Trimmomatic: a flexible trimmer for Illumina sequence data. Bioinformatics. 2014:30:2114-20.

61. Song L, Florea L, Langmead B. Lighter: fast and memory-efficient sequencing error correction without counting. Genome Biol. 2014;15:509.

62. Hackl T, Hedrich R, Schultz J, Förster F. Proovread: large-scale high-accuracy PacBio correction through iterative short read consensus. Bioinformatics. 2014;30:3004-11.

63. Xia C, Wang M, Yin C, Cornejo OE, Hulbert S, Chen X. Genome sequence resources for the wheat stripe rust pathogen (Puccinia striiformis f. Sp. tritici) and the barley stripe rust pathogen (Puccinia striiformis f. Sp. hordei). Mol Plant-Microbe Interact. 2018; https://doi.org/10.1094/MPMI-04-18-0107-A.

64. Xia C, Wang M, Cornejo OE, Jiwan DA, See DR, Chen X. Secretome characterization and correlation analysis reveal putative pathogenicity mechanisms and identify candidate avirulence genes in the wheat stripe rust fungus Puccinia striiformis f. Sp. tritici. Front Microbiol. 2017:8:2394.

65. Marçais G, Kingsford C. A fast, lock-free approach for efficient parallel counting of occurrences of k-mers. Bioinformatics. 2011;27:764-70.

66. Lander ES, Waterman MS. Genomic mapping by fingerprinting random clones: a mathematical analysis. Genomics. 1988;2:231-9.

67. DePristo MA, Banks E, Poplin R, Garimella KV, Maguire JR, Hartl C, et al. A framework for variation discovery and genotyping using next-generation DNA sequencing data. Nature Genet. 2011;43:491-8.

68. Li H, Durbin R. Fast and accurate long-read alignment with burrowswheeler transform. Bioinformatics. 2010;26:589-95.

69. Li H. A statistical framework for SNP calling, mutation discovery, association mapping and population genetical parameter estimation from sequencing data. Bioinformatics. 2011;27:2987-93.

70. Cingolani P, Platts A, Wang LL, Coon M, Nguyen $T$, Wang $L$, et al. A program for annotating and predicting the effects of single nucleotide polymorphisms, SnpEff: SNPs in the genome of Drosophila melanogaster strain w1118; iso-2; iso-3. Fly. 2012;6:80-92.

71. Kurtz S, Phillippy A, Delcher AL, Smoot M, Shumway M, Antonescu C, et al. Versatile and open software for comparing large genomes. Genome Biol. 2004;5:R12.

72. Krzywinski M, Schein J, Birol I, Connors J, Gascoyne R, Horsman D, et al. Circos: an information aesthetic for comparative genomics. Genome Res. 2009;19:1639-45.

73. Emms DM, Kelly S. OrthoFinder: solving fundamental biases in whole genome comparisons dramatically improves orthogroup inference accuracy. Genome Biol. 2015;16:157.

74. Pertea M, Pertea GM, Antonescu CM, Chang T-C, Mendell JT, Salzberg SL. StringTie enables improved reconstruction of a transcriptome from RNA-seq reads. Nature Biotechnol. 2015:33:290-5.

75. Kampstra P. Beanplot: A boxplot alternative for visual comparison of distributions. 2008. 28:Code Snippet 1. http://www.stat.cmu.edu/ rnugent/ PCMI2016/papers/Beanplot.pdf.

76. Wickham H. ggplot2: elegant graphics for data analysis. Springer; 2016. http://www.springer.com/us/book/9780387981413

77. Kanehisa M, Sato Y, Morishima K. BlastKOALA and GhostKOALA: KEGG tools for functional characterization of genome and metagenome sequences. J Mol Biol. 2016:428:726-31.

78. Al-Shahrour F, Minguez P, Tárraga J, Medina I, Alloza E, Montaner D, et al. FatiGO+: a functional profiling tool for genomic data. Integration of functional annotation, regulatory motifs and interaction data with microarray experiments. Nucleic Acids Res. 2007;35:W91-W6.

79. Sievers F, Wilm A, Dineen D, Gibson TJ, Karplus K, Li W, et al. Fast, scalable generation of high-quality protein multiple sequence alignments using Clustal omega. Mol Syst Biol. 2011;7:539.

80. Ronquist F, Teslenko M, van Der Mark P, Ayres DL, Darling A, Höhna S, et al. MrBayes 3.2: efficient Bayesian phylogenetic inference and model choice across a large model space. Syst Biol. 2012;61:539-42. 
81. Darriba D, Taboada GL, Doallo R, Posada D. ProtTest 3: fast selection of bestfit models of protein evolution. Bioinformatics. 2011;27:1164-5.

82. Sanderson MJ. r8s: inferring absolute rates of molecular evolution and divergence times in the absence of a molecular clock. Bioinformatics. 2003;19:301-2.

83. Taylor JW, Berbee ML. Dating divergences in the fungal tree of life: review and new analyses. Mycologia. 2006;98:838-49.

84. Hovmøller MS, Justesen AF. Rates of evolution of avirulence phenotypes and DNA markers in a northwest European population of Puccinia striiformis f. Sp. tritici. Mol Ecol. 2007;16:4637-47.

85. Yang Z, Nielsen R. Estimating synonymous and nonsynonymous substitution rates under realistic evolutionary models. Mol Biol Evol. 2000;17:32-43.

86. Yang Z. PAML 4: phylogenetic analysis by maximum likelihood. Mol Biol Evol. 2007;24:1586-91.

87. Suyama M, Torrents D, Bork P. PAL2NAL: robust conversion of protein sequence alignments into the corresponding codon alignments. Nucleic Acids Res. 2006;34:W609-W12.

88. Felsenstein J. \{PHYLIP\}(Phylogeny Inference Package) version 3.6 a3. 2002. http://www0.nih.go.jp/ jun/research/phylip/main.html.

89. Sperschneider J, Dodds PN, Taylor JM, Duplessis S. Computational methods for predicting effectors in rust pathogens. Wheat Rust Diseases Springer. 2017:73-83.

90. Sperschneider J, Williams AH, Hane JK, Singh KB, Taylor JM. Evaluation of secretion prediction highlights differing approaches needed for oomycete and fungal effectors. Front Plant Sci. 2015;6:1168.

91. Yin Y, Mao X, Yang J, Chen X, Mao F, dbCAN XY. A web resource for automated carbohydrate-active enzyme annotation. Nucleic Acids Res. 2012;40:W445-W51.

92. Wolf T, Shelest V, Nath N, Shelest E. CASSIS and SMIPS: promoter-based prediction of secondary metabolite gene clusters in eukaryotic genomes. Bioinformatics. 2015;32:1138-43.

93. Saier MH, Reddy VS, Tsu BV, Ahmed MS, Li C, Moreno-Hagelsieb G. The transporter classification database (TCDB): recent advances. Nucleic Acids Res. 2016;44:D372-D9.

94. Rawlings ND, Barrett AJ, Finn R. Twenty years of the MEROPS database of proteolytic enzymes their substrates and inhibitors. Nucleic Acids Res. 2015;44:D343-D50

95. Simão FA, Waterhouse RM, loannidis P, Kriventseva EV, Zdobnov EM BUSCO: assessing genome assembly and annotation completeness with single-copy orthologs. Bioinformatics. 2015;31:3210-2.

\section{Ready to submit your research? Choose BMC and benefit from:}

- fast, convenient online submission

- thorough peer review by experienced researchers in your field

- rapid publication on acceptance

- support for research data, including large and complex data types

- gold Open Access which fosters wider collaboration and increased citations

- maximum visibility for your research: over $100 \mathrm{M}$ website views per year

At BMC, research is always in progress.

Learn more biomedcentral.com/submissions 